\title{
Early Restoration of Shank3 Expression in Shank3 Knock-Out Mice Prevents Core ASD-Like Behavioral Phenotypes
}

\author{
Thomas C. Jaramillo, ${ }^{1}$ Zhong Xuan, ${ }^{2}$ Jeremy M. Reimers, ${ }^{1}{ }^{\circledR C h r i s t i n e ~ O . ~ E s c a m i l l a, ~}{ }^{1}$ Shunan Liu, ${ }^{1}$ and \\ (D) Craig M. Powell ${ }^{2,3}$
}

https://doi.org/10.1523/ENEURO.0332-19.2020

${ }^{1}$ Department of Neurology and Neurotherapeutics, University of Texas Southwestern Medical Center, Dallas, TX 75390-8813, ${ }^{2}$ Department of Neurobiology, University of Alabama at Birmingham, Birmingham, AL, and ${ }^{3}$ Civitan International Research Center at UAB, Birmingham, AL 35233

\begin{abstract}
Several genes are associated with increased risk for autism spectrum disorder (ASD), neurodevelopmental disorders that present with repetitive movements and restricted interests along with deficits in social interaction/communication. While genetic alterations associated with ASD are present early in life, ASD-like behaviors are difficult to detect in early infancy. This raises the issue of whether reversal of an ASDassociated genetic alteration early in life can prevent the onset of ASD-like behaviors. Genetic alterations of SHANK3, a well-characterized gene encoding a postsynaptic scaffolding protein, are estimated to contribute to $\sim 0.5 \%$ of ASD and remain one of the more replicated and well-characterized genetic defects in ASD. Here, we investigate whether early genetic reversal of a Shank3 mutation can prevent the onset of ASD-like behaviors in a mouse model. Previously, we have demonstrated that mice deficient in Shank3 display a wide range of behavioral abnormalities such as repetitive grooming, social deficits, anxiety, and motor abnormalities. In this study, we replicate many of these behaviors in Shank3 mutant mice. With early genetic restoration of wild-type (WT) Shank3, we rescue behaviors including repetitive grooming and social, locomotor, and rearing deficits. Our findings support the idea that the underlying mechanisms involving ASD behaviors in mice deficient in Shank3 are susceptible to early genetic correction of Shank3 mutations.
\end{abstract}

Key words: autism; autism spectrum disorder; behavior; genetic reversal; Phelan-McDermid syndrome; Shank3

\section{Significance Statement}

Rare, de novo, single gene copy number variants and mutations are known causes of autism. The SHANK3 gene is among the most common and replicated genetic causes of autism. With the advent of gene therapy, interest is growing in understanding whether genetic animal models of autism can have their phenotypes ameliorated by genetic reversal. This study confirms that early genetic restoration of a Shank3 mutant mouse model can ameliorate behavioral symptoms with face validity for autism.

\footnotetext{
Received August 19, 2019; accepted April 9, 2020; First published April 17, 2020.

C.M.P. has accepted travel funds and honoraria to speak once at each of the following companies: Psychogenics, Inc.; Astra-Zeneca; Roche; Pfizer; and Dainippon Sumitomo Pharma Co. and also has investigator-initiated grant funding for clinical research with Novartis. None of these activities represents a competing interest to the current study. All other authors declare no competing financial interests.
}

Author contributions: T.C.J., C.O.E., and C.M.P. designed research; T.C.J., Z.X., J.M.R., C.O.E., and S.L. performed research; Z.X. and C.M.P. contributed unpublished reagents/analytic tools; T.C.J. and C.O.E. analyzed data; T.C.J. and C.M.P. wrote the paper. 


\section{Introduction}

The Shank3 gene encodes a multi-domain, scaffolding protein located at the postsynaptic density of excitatory synapses that interacts with a number of scaffolding and signaling proteins to form complexes that ensure proper synaptic formation and function (Naisbitt et al., 1999; Tu et al., 1999; Ebert and Greenberg, 2013). Approximately $0.5-1 \%$ of all individuals with autism spectrum disorder (ASD) have SHANK3 mutations (Jiang and Ehlers, 2013). Loss-of-function of one copy of SHANK3 is a known cause of Phelan-McDermid syndrome (PMS), a cause of ASD and intellectual disability (Phelan, 2008; Costales and Kolevzon, 2015; Tachibana et al., 2017; Mitz et al., 2018). ASD is characterized by the presentation of two core phenotypes, deficits in social interaction/communication and restricted interests and repetitive behaviors. In patients, symptoms of ASD can be evident as early as 12 months, while actual diagnosis of these symptoms as ASD may not occur until later in childhood. In this study we address the possibility of early genetic intervention to prevent the onset of ASD-like behaviors.

Previously, we generated and characterized a mouse model deficient in a subset of major isoforms of Shank3 (Shank $3^{\mathrm{E} 13}$ ) that behaviorally displayed excessive grooming, social interaction deficits, decreased rearing, locomotor deficits, and learning and memory deficits (Jaramillo et al., 2017). Additionally, other studies in similar Shank3 mouse models have replicated these findings in addition to observing decreased hippocampal long-term potentiation, morphologic and structural abnormalities like reduced spine density and longer dendritic length, along with a reduction in synaptic proteins that associate with SHANK3 (Bozdagi et al., 2010; Peça et al., 2011; Wang et al., 2011; Yang et al., 2012; Kouser et al., 2013). One elegant study also examined the ability of adult and early developmental genetic reversal to rescue phenotypes in a Shank3 mutant mouse model (Mei et al., 2016). In efforts parallel to those of Guoping Feng's laboratory (Mei et al., 2016), we also generated a similar, reversible Shank3 mouse model to examine the question of developmental reversibility of phenotypes.

Our Shank $3^{\mathrm{E} 13}$ mouse model was generated by inserting a transcriptional "stop" (neo-stop) cassette flanked by loxP sites into the intron before the start of exon 13, the first exon encoding the PDZ domain [postsynaptic density-95 (PSD-95)/disk large (DLG)/zona occludens-1 (ZO-

This work was supported by National Institutes of Health Grants R01HD069560 and R01HD069560-S1 (to C.M.P.), Autism Speaks (C.M.P.), an Autism Science Foundation Pre-doctoral Fellowship (C.O.E.), The Hartwell Foundation (C.M.P.), Ed and Sue Rose Distinguished Professorship in Neurology (C.M.P.), and gifts from Dr. Clay Heighten and Dr. Debra Caudy and BRAINS for Autism (C.M.P.).

T. C. Jaramillo's present address: Rodent Behavioral Core, Lerner Research Institute, Cleveland 44195, OH.

Correspondence should be addressed to Craig M. Powell at craigpow@ uab.edu.

https://doi.org/10.1523/ENEURO.0332-19.2020

Copyright (C) 2020 Jaramillo et al.

This is an open-access article distributed under the terms of the Creative Commons Attribution 4.0 International license, which permits unrestricted use, distribution and reproduction in any medium provided that the original work is properly attributed.
1)]. Because of the flanking loxP sites, the Shank $3^{\mathrm{E} 13}$ model can be genetically reversed to wild-type (WT) by removal of the neo-stop cassette on expression of Cre-recombinase. Our model is similar to the one generated in Feng's laboratory in that both induce Shank3 gene expression at its endogenous genomic locus ensuring that SHANK3 expression is within its physiologic concentrations. However, while Feng's model uses tamoxifen for Shank3 induction which leads to some toxicity including weight loss in WT mice, we used a 2-transgene system involving TTA and Cre expression in an attempt to temporally regulate Shank3 expression. Two transgenic mouse lines, tetracycline-controlled activator protein under control of the neuron-specific enolase promoter (NSE-tTA) and Cre recombinase under the control of a tetracyclineresponse promoter element (tetO; tetO-Cre) were bred with the Shank3 ${ }^{\mathrm{E} 13}$ mutant mouse model. The NSE-tTA transgene leads to expression of tTA at approximately embryonic day (E)18 in mice (Forss-Petter et al., 1990; Alouani et al., 1993). tTA expression activates the tetO promoter to drive Cre-recombinase expression, allowing for recombination of the premature stop cassette between the flanking loxP sites (i.e., genetic reversal). Previous reversal studies in Shank3 mouse models of ASD (Mei et al., 2016) focused on restoration of SHANK3 expression in homozygous knock-out (KO) mice. In this study, however, we include the heterozygous Shank3 mice to provide more accurate construct validity to the human condition. While we had planned to examine both adult and early genetic restoration, we are unable to prevent restoration of Shank3 using doxycycline in our model. Following early genetic reversal, Shank $3^{\mathrm{E} 13}$ mutant mice express WT Shank3 and no longer display repetitive grooming or social deficits. As a control and replication of our previous findings, these atypical behaviors were observed in Shank $3^{\mathrm{E} 13}$ mutant mice expressing only one of the two transgenes. These results replicate many, but not all, of our original findings in the Shank3 ${ }^{\mathrm{E} 13}$ mutant mice and confirm that some behaviors with face validity for ASD remain responsive to re-expression of WT Shank3 early in development. Our findings suggest early genetic reversal as a potential treatment for ASD-like behaviors associated with Shank3 deficiency.

\section{Materials and Methods}

\section{Generation of genetically reversible Shank $3^{\mathrm{E} 13}$ mutant mice}

The generation of the Shank $3^{\mathrm{E} 13}$ mutant mice is described in our previous publication (Jaramillo et al., 2017). Briefly, a neo-stop targeting construct was designed by combining PGK-neo gene cassette with the His3-SV40 pA sequences and inserted into a unique Bglll site located within intron 12, after exon 12 of the mouse Shank3 gene (Dragatsis and Zeitlin, 2001; Guy et al., 2007). The neostop cassette prevents transcription of Shank3 beyond exon 12. The neo-stop cassette is flanked by loxP sites making this insertion reversible in the presence of cre-recombinase. Following verification of proper insertion, Shank3 ${ }^{\mathrm{E} 13}$ mice were used to generate two lines of mice. 
One line consisted of heterozygous Shank ${ }^{\mathrm{E} 13}$ mice bred with NSE-tTA (tetracycline-regulated activator protein under the control of neuron specific enolase promoter; The Jackson Laboratory stock \#003767). Although the description for the NSE-tTA mouse line from The Jackson Laboratory and a publication (Chen et al., 1998) suggest selective expression of TTA in the striatum and cerebellum, our studies revealed global expression of tTA under our specific conditions (Fig. 1C). To determine whether a mouse was positive for the NSE-tTA transgene, two sets of primers were used. Internal primers for the transgene, forward 5'-CAAATGTTGCTTGTCTGGTG-3' and reverse 5'-GTCAGTCGAGTGCACAGTTT-3', produced a 200-bp band. Primers for the transgene, forward 5'-CGCTGTGGGGCATTTTACTTTAG-3' and reverse 5'CATGTCCAGATCGAAATCGTC-3' produced a 450-bp band for a second confirmation.

A second mouse line consisted of heterozygous Shank $3^{\mathrm{E} 13}$ mice bred with tetO-Cre (cre-recombinase under control of a tetracycline-response promoter element, tetO; The Jackson Laboratory stock \#003767). To verify the presence of the transgene, forward 5'-CGCTGTGGGGCATTTACTTTAG-3' and reverse 5'-CATGTCCAGATCGAAATCGTC- $3^{\prime}$ primers produced a 100-bp band, confirming presence of the tetOCre transgene. A pair of internal primers was also used; forward 5'-CAAATGTTGCTTGTCTGGTG-3' and reverse 5'GTCAGTCGAGTGCACAGTTT-3' produced a 200-bp band. Finally, the two mouse lines generated (Shank3 ${ }^{\text {E13/NSE-tTA }}$ mice and Shank $3^{\mathrm{E} 13 / \mathrm{tetO}-\mathrm{Cre}}$ mice) were bred together to gen-

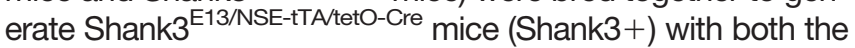
tTA and tetO-cre transgenes and either WT, heterozygous, or homozygous for the Shank $3^{\mathrm{E} 13}$ allele. Mice that only retained one of the transgenes were used as controls (Shank3-) and remained mutant for Shank3. It is worth noting that our attempts to use doxycycline to suppress cre-recombinase expression in this particular model were unsuccessful. Thus, we focused on the early developmental rescue of Shank3 gene expression in the absence of doxycycline.

\section{Behavioral overview}

All mice tested were age- and sex-matched littermate progeny of matings between mice heterozygous for Shank3 ${ }^{\mathrm{E} 13}$ with floxed neo-stop cassette and one of the regulator transgenes, NSE-tTA or tetO-cre. Shank3 ${ }^{\mathrm{E} 13}$ with both regulator transgenes constituted the experimental cohort and are referred to as Shank3+ mice, while Shank $3^{\mathrm{E} 13}$ mice with only one of the two regulator transgenes constituted the control cohort and are referred to as Shank3- (WT-, HET-, KO-). Genetic background is consistent among all mice due to the use of a single breeding strategy to produce all experimental offspring from the same cross. An experimenter blind to genotypes performed all behavioral tests. The behavioral testing consisted of two cohorts. The first included mice WT+, heterozygous $+(\mathrm{HET}+)$, and homozygous $+(\mathrm{KO}+)$ for floxed, neo-stop-cassette-containing Shank $3^{\mathrm{E} 13}$ expressing both transgenes (tetOp-cre and NSE-tTA). The second cohort included WT-, HET-, and KO- Shank $3^{\mathrm{E} 13}$ mice with only one of the two transgenes (tetO-cre or NSEtTA). Cohort 1 consisted of $n=22$ WT (WT,+ 10 male and
12 female), $n=17$ heterozygous (HET+, eight males and nine females), $n=14 \mathrm{KO}$ (KO+, eight males and six females) mice, while cohort 2 consisted of $n=27$ WT (WT-, 16 male and 11 female), $n=20$ heterozygous (HET-, 10 males and 10 females), $n=19 \mathrm{KO}$ (KO-, 10 males and 9 females) mice.

Behaviors were tested in the following order: elevated plus maze, dark/light, open field (OF), locomotor, rearing, grooming, three-box social interaction, caged conspecific (also called social interaction with a caged adult), marble burying, rotarod, social interaction with free moving juvenile mouse, genotype-sex-matched adult social interaction, olfactory "cookie-finding" palatable treat test, nesting, cue and contextual fear conditioning, and Morris water maze. Behavioral results are presented in a different order than tested to ease the flow of presentation. Analysis of behavioral data were conducted using StatPlus software (version 2015, AnalystSoft) using either two-way ANOVA or threeway repeated measures ANOVA with genotype and sex as the main variables and trial, bouts, or time as the repeated measure where applicable. Post hoc planned comparisons were applied for significant effects and interactions. For detailed statistical results, see Table 1.

\section{Behavioral tests}

\section{Elevated plus maze}

This test was conducted as described previously (Etherton et al., 2009). Briefly, mice were placed in the center of the maze (each arm was $30 \mathrm{~cm}$ long and $5 \mathrm{~cm}$ wide with $25-\mathrm{cm}$-high walls on the closed arms) and allowed to freely explore for $5 \mathrm{~min}$. All mice were tested under dim white light at $\sim 7$ lux. Noldus Ethovision version 3.1 was used to track and record mouse behavior.

\section{Locomotor and rearing activity}

Locomotor activity was measured as described previously (Powell et al., 2004; Tabuchi et al., 2007; Etherton et al., 2009). Mice were placed in novel cages (a clean cage with the same dimensions as their home cage; $\mathrm{L} \times \mathrm{W} \times$ $\mathrm{H}=27.3 \times 16.5 \times 12.7 \mathrm{~cm}$ ) with minimal bedding and allowed to freely explore for $2 \mathrm{~h}$ under red lighting. Horizontal locomotor activity (i.e., the number of photobeam breaks) was measured by computer software (San Diego Instruments) and data were analyzed in 5-min bins.

\section{Dark/light test}

The dark/light test was conducted as described previously (Powell et al., 2004; Blundell et al., 2009). Mice were placed in the dark chamber (each chamber was $25 \times 26 \mathrm{~cm}$ with 2066 lux on the light side and $\sim 1$ lux on the dark side) and allowed to habituate for 2 min. After habituation, mice were allowed to freely explore both chambers for $10 \mathrm{~min}$.

\section{Rotarod}

The rotarod test was conducted as described previously (Powell et al., 2004). Briefly, mice were placed on a stationary rotarod (IITC Life Science) that was then activated and accelerated from 0 to $45 \mathrm{rpm}$ over $5 \mathrm{~min}$. The latency for the mice to fall off the rod was measured. If a mouse held onto the rotating rod for one complete 
Table 1: Statistical analysis

\begin{tabular}{|c|c|c|c|c|}
\hline $\begin{array}{l}\text { Shank3- }(n=11 \\
\text { WT, } 8 \text { HET, } 7 \\
\text { KO pairs) }\end{array}$ & $\begin{array}{l}\text { Genotype sex } \\
\text { match }\end{array}$ & $\begin{array}{l}\text { Interaction } \\
\text { time (s) }\end{array}$ & $\begin{array}{l}\text { Genotype and sex } \\
\text { Post hoc: Scheffe geno- } \\
\text { type } \\
\text { Fig. } 2 A\end{array}$ & 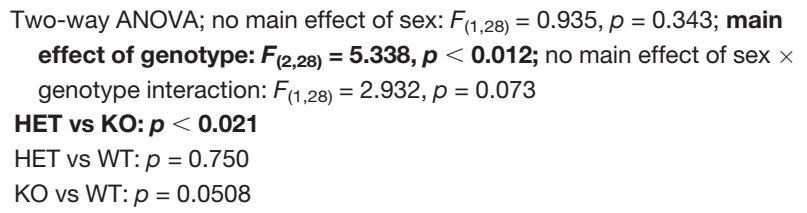 \\
\hline $\begin{array}{l}\text { Shank3+ }(n=11 \\
\text { WT, } 8 \text { HET, } 7 \\
\text { KO pairs })\end{array}$ & & $\begin{array}{l}\text { Interaction } \\
\text { time (s) }\end{array}$ & $\begin{array}{l}\text { Genotype and sex } \\
\text { Fig. } 2 B\end{array}$ & $\begin{array}{l}\text { Two-way ANOVA; no main effect of sex: } F_{(1,25)}=0.733, p=0.401 \text {; no } \\
\text { main effect of genotype: } F_{(2,25)}=0.098, p=0.906 ; \text { no main effect of } \\
\text { sex } \times \text { genotype interaction: } F_{(1,25)}=0.517, p=0.604\end{array}$ \\
\hline $\begin{array}{l}\text { Shank3- }(n=24 \\
\text { WT, } 16 \text { HET, } \\
20 \mathrm{KO})\end{array}$ & $\begin{array}{c}\text { Social interaction } \\
\text { with a juvenile }\end{array}$ & $\begin{array}{l}\text { Interaction } \\
\text { time (s) }\end{array}$ & $\begin{array}{l}\text { Sex, genotype, trial } \\
\text { Post hoc: pairwise } t \text { test: } \\
\quad \text { initial vs recognition } \\
\text { Fig. } 2 C\end{array}$ & 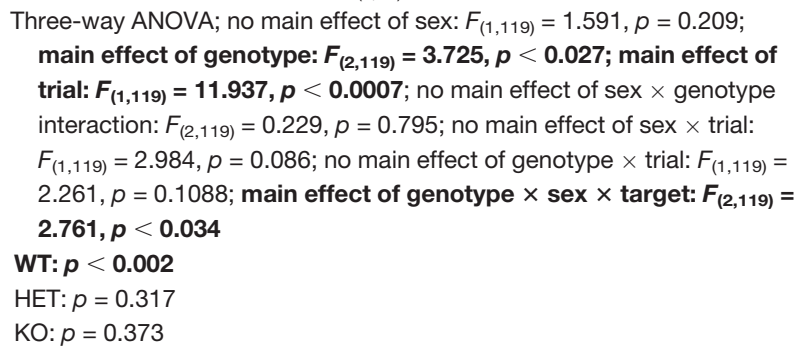 \\
\hline $\begin{array}{l}\text { Shank3+ }(n=24 \\
\text { WT, } 19 \text { HET, } \\
16 \mathrm{KO})\end{array}$ & & $\begin{array}{l}\text { Interaction } \\
\text { time (s) }\end{array}$ & $\begin{array}{l}\text { Sex, genotype, trial } \\
\text { Post hoc: pairwise } t \text { test: } \\
\text { initial vs recognition } \\
\text { Fig. } 2 D\end{array}$ & 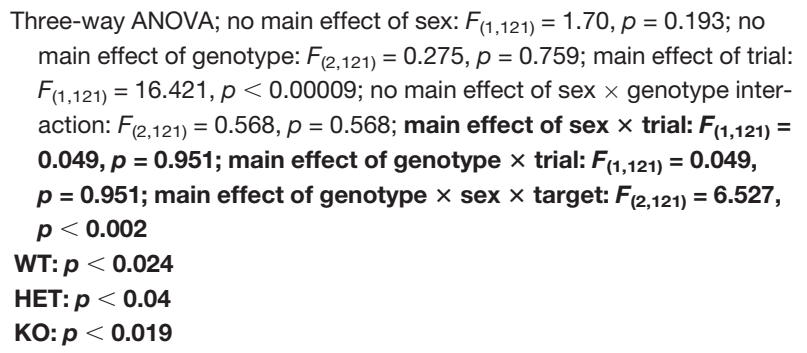 \\
\hline $\begin{array}{l}\text { Shank3- }(n=24 \\
\text { WT, } 16 \text { HET, } \\
20 \mathrm{KO})\end{array}$ & & $\begin{array}{l}\text { Trial 1: chamber } \\
\text { bias }\end{array}$ & $\begin{array}{l}\text { Genotype and chamber } \\
\text { Post hoc: Scheffe; sex } \times \\
\text { chamber } \\
\text { Fig. } 2 E\end{array}$ & $\begin{array}{l}\text { Three-way ANOVA; no main effect of sex: } F_{(1,126)}=0.539, p=0.464 \text {; no } \\
\text { main effect of genotype: } F_{(2,126)}=0.115, p=0.890 \text {; no main effect of } \\
\text { chamber: } F_{(1,126)}=0.362, p=0.548 ; \text { no main effect of sex } \times \text { genotype } \\
\text { interaction: } F_{(2,126)}=0.676, p=0.510 ; \text { main effect of sex } \times \text { chamber: } \\
\boldsymbol{F}_{(\mathbf{1}, 126)}=\mathbf{1 6 . 4 2 , p}<\mathbf{0 . 0 0 0 0 9} \text {; main effect of genotype } \times \text { chamber: } \\
\boldsymbol{F}_{(\mathbf{1}, 126)}=, \boldsymbol{p}<\mathbf{0 . 0 4 3} \text {; no main effect of genotype } \times \text { sex } \times \text { chamber: } \\
F_{(2,126)}=0.676, p=0.510 \\
\text { Back: } p=0.117 \\
\text { Front: } p=0.294\end{array}$ \\
\hline & & $\begin{array}{l}\text { Trial 2: duration in } \\
\text { chamber; in- } \\
\text { animate vs } \\
\text { social }\end{array}$ & $\begin{array}{l}\text { Genotype and interaction } \\
\text { target (social vs inani- } \\
\text { mate) } \\
\text { Post hoc: Scheffe } \\
\text { chamber: mouse vs inani- } \\
\text { mate } \\
\text { Sex } \times \text { chamber } \\
\text { Fig. } 2 F\end{array}$ & 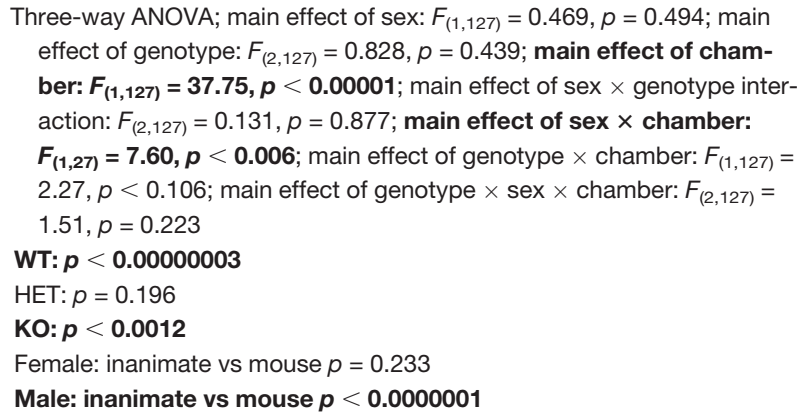 \\
\hline & & $\begin{array}{l}\text { Trial 3: duration in } \\
\text { chamber; fa- } \\
\text { miliar vs novel }\end{array}$ & $\begin{array}{l}\text { Sex, genotype, and cham- } \\
\text { ber } \\
\text { Post hoc Scheffe } \\
\text { chamber: novel vs familiar } \\
\text { Fig. } 2 G\end{array}$ & $\begin{array}{l}\text { Three-way ANOVA; main effect of sex: } F_{(1,122)}=0.472, p=0.493 \text {; main } \\
\text { effect of genotype: } F_{(2,122)}=0.150, p=0.860 ; \text { main effect of cham- } \\
\text { ber: } \boldsymbol{F}_{(\mathbf{1}, 122)}=\mathbf{2 . 2 4 2 ,} \boldsymbol{p}<\mathbf{0 . 0 1 3 7} \text {; } \text { main effect of sex } \times \text { genotype inter- } \\
\text { action: } F_{(2,122)}=0.070, p=0.924 ; \text { main effect of sex } \times \text { chamber: } \\
F_{(1,122)}=3.54, p=0.062 ; \text { main effect of genotype } \times \text { chamber: } F_{(1,122)}= \\
2.816, p=0.064 ; \text { main effect of genotype } \times \text { sex } \times \text { chamber: } \\
\boldsymbol{F}_{(\mathbf{2}, \mathbf{1 2 2})}=\mathbf{0 . 0 4 9 , p = 0 . 9 5 1} \\
\text { WT: } \boldsymbol{p}<\mathbf{0 . 0 0 3} \\
\text { HET: } p=0.439 \\
\text { KO: } p=0.442\end{array}$ \\
\hline
\end{tabular}


Table 1: Continued

\begin{tabular}{|c|c|c|c|}
\hline \multirow[t]{2}{*}{$\begin{array}{l}\text { Shank3+ }(n=24 \\
\text { WT, } 19 \text { HET, } \\
20 \mathrm{KO})\end{array}$} & $\begin{array}{l}\text { 3-Box social } \\
\text { interaction }\end{array}$ & $\begin{array}{l}\text { Trial 1: chamber } \\
\text { bias }\end{array}$ & $\begin{array}{l}\text { Genotype and chamber } \\
\text { Fig. } 2 \mathrm{H}\end{array}$ \\
\hline & & $\begin{array}{l}\text { Trial 2: duration in } \\
\text { chamber; in- } \\
\text { animate vs } \\
\text { social }\end{array}$ & $\begin{array}{l}\text { Genotype and interaction } \\
\text { target (social vs inani- } \\
\text { mate) } \\
\text { Post hoc: Scheffe; cham- } \\
\text { ber: mouse vs inanimate } \\
\text { Fig. } 2 \text { I }\end{array}$ \\
\hline
\end{tabular}
Shank3- $(n=24 \quad$ Caged
WT, 16 HET,
20 KO $)$

Shank3+ $(n=24$

WT, 19 HET,

$16 \mathrm{KO})$
Interaction time

Sex, genotype, target Fig. $2 L$

\section{Shank3- $(n=24$ WT, 16 HET, $20 \mathrm{KO})$}

Interaction time

Sex, genotype, target Fig. $2 K$

Trial 3: duration in Sex, genotype, and chamchamber; fa- ber miliar vs novel Post hoc: Scheffe; chamber: novel vs familiar Fig. 2J

\section{Duration}

Grooming

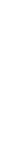

Bouts

Duration

Shank3+ $(n=24$

WT, 19 HET,

$16 \mathrm{KO})$

Shank3- $(n=24$

Open field

WT, $16 \mathrm{HET}$,

$20 \mathrm{KO})$

Bouts Sex and genotype Fig. $3 D$
Three-way ANOVA; main effect of sex: $F_{(1,81)}=3.306, p=0.073$; main effect of genotype: $F_{(2,81)}=0.071, p=0.930$; main effect of chamber: $F_{(1,81)}=0.059, p=0.808$; main effect of sex $\times$ genotype interaction: $F_{(2,81)}=0.134, p=0.874$; main effect of sex $\times$ chamber: $F_{(1,81)}=$ $0.001, p=0.965$; main effect of genotype $\times$ chamber: $F_{(1,81)}=$, $p=0.042$; main effect of genotype $\times$ sex $\times$ chamber: $F_{(2,81)}=0.784$, $p=0.460$

Three-way ANOVA; no main effect of sex: $F_{(1,87)}=0.313, p=0.577$; no main effect of genotype: $F_{(2,87)}=1.121, p=0.300$; main effect of chamber: $F_{(1,87)}=\mathbf{1 5 . 3 2 7 , p}<\mathbf{0 . 0 0 0 2}$; no main effect of sex $\times$ genotype interaction: $F_{(2,87)}=0.067, p=0.934$; no main effect of sex $\times$ chamber: $F_{(1,87)}=0.940, p=0.335$; no main effect of genotype $\times$ chamber: $F_{(1,87)}=0.446, p=0.641$; no main effect of genotype $\times$ sex $\times$ chamber: $F_{(2,87)}=2.049, p=0.135$

WT: $\boldsymbol{p}<\mathbf{0 . 0 0 4 4}$

HET: $p=0.153$

KO: $p<0.0012$

Three-way ANOVA; no main effect of sex: $F_{(1,85)}=0.175, p=0.676$; no main effect of genotype: $F_{(2,85)}=0.354, p=0.703$; main effect of chamber: $\boldsymbol{F}_{(\mathbf{1}, 85)}=\mathbf{1 4 . 7 5}, p<\mathbf{0 . 0 0 0 2}$; no main effect of sex $\times$ genotype interaction: $F_{(2,85)}=0.803, p=0.451$; no main effect of sex $\times$ chamber: $F_{(1,85)}=0.0021, p=0.963$; no main effect of genotype $\times$ chamber: $F_{(1,85)}=0.451, p=0.638$; main effect of genotype $\times$ sex $\times$ chamber: $F_{(2,85)}=4.321, p<0.016$

WT: $\boldsymbol{p}<\mathbf{0 . 0 1 7}$

HET: $p=0.099$

KO: $\boldsymbol{p}<\mathbf{0 . 0 3 7}$

Three-way ANOVA; no main effect of sex: $F_{(1,122)}=0.720, p=0.397$; no main effect of genotype: $F_{(2,122)}=0.652, p=0.522$; main effect of tar-

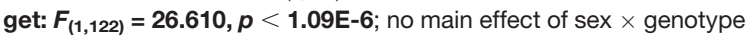
interaction: $F_{(2,122)}=0.218, p=0.804$; no main effect of sex $\times$ target: $F_{(1,122)}=1.029, p=0.312$; no main effect of genotype $\times$ target: $F_{(1,122)}=0.759, p=0.470$; no main effect of genotype $\times \operatorname{sex} \times$ target: $F_{(2,122)}=1.730, p=0.182$

Three-way ANOVA; no main effect of sex: $F_{(1,107)}=0.004, p=0.945$; no main effect of genotype: $F_{(2,107)}=0.785, p=0.458$; main effect of target: $\boldsymbol{F}_{(1,107)}=\mathbf{6 . 5 5 4 , p}<\mathbf{0 . 0 1 2}$; no main effect of sex $\times$ genotype interaction: $F_{(2,107)}=0.042, p=0.958$; no main effect of sex $\times$ target: $F_{(1,107)}=0.737, p=0.392$; no main effect of genotype $\times$ target: $F_{(1,107)}=0.475, p=0.623$; no main effect of genotype $\times \operatorname{sex} \times$ target: $F_{(2,107)}=0.085, p=0.918$

Two-way ANOVA; no main effect of sex: $F_{(1,62)}=1.638, p=0.205$; main effect of genotype: $\boldsymbol{F}_{(2,62)}=\mathbf{1 1 . 8 4 4 , p}<\mathbf{0 . 0 0 0 0 5}$; no sex $\times$ genotype interaction: $F_{(1,62)}=1.347, p=0.267$

WT vs HET: $\boldsymbol{p}<\mathbf{0 . 0 0 0 4 5}$

HET vs KO: $p=0.968$

WT vs KO: $\boldsymbol{p}<\mathbf{0 . 0 0 0 7}$

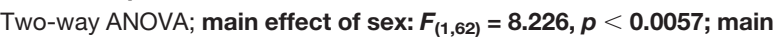
effect of genotype: $\boldsymbol{F}_{(2,62)}=\mathbf{3 . 7 3 0 , p}<\mathbf{0 . 0 3 0}$; no main effect of sex $\times$ genotype interaction: $F_{(1,62)}=0.750, p=0.476$

WT vs HET: $p=0.666$

HET vs KO: $p=0.265$

WT vs KO: $\boldsymbol{p}<\mathbf{0 . 0 3 0}$

Two-way ANOVA; no main effect of sex: $F_{(1,60)}=0.015, p=0.901$; no main effect of genotype: $F_{(2,60)}=0.558, p=0.575$; no main effect of sex $\times$ genotype interaction: $F_{(1,60)}=1.15, p=0.323$

Two-way ANOVA; no main effect of sex: $F_{(1,60)}=0.0975, p=0.755$; no main effect of genotype: $F_{(2,60)}=0.398, p=0.673$; no main effect of sex $\times$ genotype interaction: $F_{(1,60)}=0.514, p=0.600$

Two-way ANOVA; no main effect of sex: $F_{(1,65)}=0.619, p=0.434$; main effect of genotype: $F_{(2,65)}=1.336, p=0.270$; no main effect of sex $\times$ genotype interaction: $F_{(1,65)}=0.177, p=0.838$ 
Table 1: Continued

\begin{tabular}{|c|c|c|c|c|}
\hline & & $\begin{array}{l}\text { Distance traveled } \\
\text { left }\end{array}$ & $\begin{array}{l}\text { Sex and genotype } \\
\text { Fig. } 3 F\end{array}$ & $\begin{array}{l}\text { Two-way ANOVA; no main effect of sex: } F_{(1,65)}=0.764, p=0.385 \text {; main } \\
\text { effect of genotype: } F_{(2,65)}=3.12, p=0.051 ; \text { sex } \times \text { genotype interac- } \\
\text { tion: } F_{(1,65)}=0.589, p=0.557\end{array}$ \\
\hline & & Thigmotaxis & $\begin{array}{l}\text { Sex and genotype } \\
\text { Fig. } 3 F\end{array}$ & $\begin{array}{l}\text { Two-way ANOVA; no main effect of sex: } F_{(1,65)}=0.986, p=0.324 \text {; main } \\
\text { effect of genotype: } F_{(2,65)}=2.59, p=0.083 ; \text { sex } \times \text { genotype interac- } \\
\text { tion: } F_{(1,65)}=0.124, p=0.882\end{array}$ \\
\hline \multirow[t]{2}{*}{$\begin{array}{l}\text { Shank3+ }(n=24 \\
\text { WT, } 19 \mathrm{HET} \\
16 \mathrm{KO})\end{array}$} & & Time in left & $\begin{array}{l}\text { Sex and genotype } \\
\text { Post hoc: Scheffe } \\
\text { Sex: female } \\
\text { Sex: male } \\
\text { Male vs female } \\
\text { Fig. } 3 G\end{array}$ & $\begin{array}{l}\text { Two-way ANOVA; no main effect of sex: } F_{(1,46)}=3.477, p<0.0402 ; \\
\quad \text { main effect of genotype: } \boldsymbol{F}_{(2,46)}=\mathbf{6 . 8 6 4 , p}<\mathbf{0 . 0 1 2} \text {; no main effect of } \\
\text { sex } \times \text { genotype interaction: } F_{(1,46)}=2.137, p=0.130 \\
\text { WT vs HET: } \boldsymbol{p}<\mathbf{0 . 0 1 4} \\
\text { HET vs KO: } p=0.067 \\
\text { WT vs KO: } p=0.983 \\
\text { WT vs HET: } p=0.973 \\
\text { HET vs KO: } p=0.984 \\
\text { WT vs KO: } p=0.911 \\
\text { WT: } p=0.710 \\
\text { HET: } p<0.001 \\
\text { KO: } p=0.864\end{array}$ \\
\hline & & Thigmotaxis & $\begin{array}{l}\text { Sex and genotype } \\
\text { Male vs female } \\
\text { Fig. } 3 \mathrm{H}\end{array}$ & $\begin{array}{l}\text { Two-way ANOVA; no main effect of sex: } F_{(1,46)}=0.038, p=0.961 \text {; } \text { main } \\
\text { effect of genotype: } F_{(2,46)}=6.729, p<0.012 ; \text { no main effect of sex } \times \\
\text { genotype interaction: } F_{(1,46)}=0.162, p=0.850 \\
\text { WT: } p=0.199 \\
\text { HET: } p=0.223 \\
\text { KO: } p=0.054\end{array}$ \\
\hline \multirow[t]{2}{*}{$\begin{array}{l}\text { Shank3- }(n=24 \\
\text { WT, } 16 \text { HET, } \\
20 \mathrm{KO})\end{array}$} & Dark-light test & $\begin{array}{l}\text { Latency to } \\
\text { explore }\end{array}$ & $\begin{array}{l}\text { Latency to explore } \\
\text { Fig. } 3 /\end{array}$ & $\begin{array}{l}\text { Two-way ANOVA; no main effect of sex: } F_{(1,50)}=2.37, p=0.130 \text {; no } \\
\quad \text { main effect of genotype: } F_{(2,50)}=0.128, p=0.879 ; \text { no main effect of } \\
\text { sex } \times \text { genotype interaction: } F_{(1,50)}=0.1 .27, p=0.288\end{array}$ \\
\hline & & $\begin{array}{l}\text { Duration in light } \\
\text { chamber }\end{array}$ & $\begin{array}{l}\text { Sex and genotype } \\
\text { Post hoc: Scheffe } \\
\text { Sex: female } \\
\text { Sex: male } \\
\text { Female vs male } \\
\text { Fig. } 3 \mathrm{~J}\end{array}$ & $\begin{array}{l}\text { Two-way ANOVA; no main effect of sex: } F_{(1,50)}=0.976, p=0.328 ; \text { main } \\
\text { effect of genotype: } \boldsymbol{F}_{(2,50)}=\mathbf{3 . 7 0 ,} \boldsymbol{p}<\mathbf{0 . 0 3 2} \text { sex } \times \text { genotype inter- } \\
\text { action: } \boldsymbol{F}_{(1,50)}=\mathbf{3 . 7 4 1}, \boldsymbol{p}<\mathbf{0 . 0 3 1} \\
\text { HET vs KO: } \boldsymbol{p}<\mathbf{0 . 0 3 2} \\
\text { HET vs WT: } p=0.300 \\
\text { KO vs WT: } p=0.395 \\
\text { HET vs KO: } \boldsymbol{p}<\mathbf{0 . 0 0 1 5} \\
\text { HET vs WT: } p=0.194 \\
\text { KO vs WT: } p=0.082 \\
\text { HET vs KO: } p=0.997 \\
\text { HET vs WT: } p=0.990 \\
\text { KO vs WT: } p=0.998 \\
\text { WT: } p=0.584 \\
\text { HET: } \boldsymbol{p}<\mathbf{0 . 0 3 5} \\
\text { KO: } p=0.067\end{array}$ \\
\hline
\end{tabular}


Table 1: Continued

\begin{tabular}{|c|c|c|c|c|}
\hline \multirow[t]{12}{*}{$\begin{array}{l}\text { Shank3+ }(n=24 \\
\text { WT,19 HET, } 16 \\
\text { KO) }\end{array}$} & Dark-light test & $\begin{array}{l}\text { Latency to } \\
\text { explore }\end{array}$ & $\begin{array}{l}\text { Sex and genotype } \\
\text { Fig. } 3 K\end{array}$ & $\begin{array}{l}\text { Two-way ANOVA; no main effect of sex: } F_{(1,47)}=0.649, p=0.424 \text {; no } \\
\text { main effect of genotype: } F_{(2,47)}=0.812, p=0.450 ; \text { no main effect of } \\
\text { sex } \times \text { genotype interaction: } F_{(1,47)}=0.396, p=0.674\end{array}$ \\
\hline & & $\begin{array}{l}\text { Duration in dark } \\
\text { chamber }\end{array}$ & $\begin{array}{l}\text { Sex and genotype } \\
\text { Post hoc: Scheffe } \\
\text { Female vs male }\end{array}$ & $\begin{array}{l}\text { Two-way ANOVA; no main effect of sex: } F_{(1,47)}=1.405, p=0.242 \text {; no } \\
\text { main effect of genotype: } F_{(2,47)}=0.177, p=0.838 \text {; no main effect of } \\
\text { sex } \times \text { genotype interaction: } F_{(1,47)}=0.183, p=0.833\end{array}$ \\
\hline & & & Female & WT: $p=0.511$ \\
\hline & & & Male & $\mathrm{HET}: p=0.862$ \\
\hline & & & Fig. $3 L$ & $\mathrm{KO}: p=0.259$ \\
\hline & & & & HET vs KO: $p=0.997$ \\
\hline & & & & HET vs WT:0.995 \\
\hline & & & & KO vs WT: $p=0.999$ \\
\hline & & & & HET vs KO: $p=0.712$ \\
\hline & & & & HET vs WT: $p=0.939$ \\
\hline & & & & KO vs WT: $p=0.872$ \\
\hline & & $\begin{array}{l}\text { Duration in light } \\
\text { chamber }\end{array}$ & $\begin{array}{l}\text { Sex and genotype } \\
\text { Fig. } 3 L\end{array}$ & $\begin{array}{l}\text { Two-way ANOVA; no main effect of sex: } F_{(1,47)}=1.40, p=0.242 \text {; no } \\
\text { main effect of genotype: } F_{(2,47)}=0.177, p=0.838 \text {; no main effect of } \\
\text { sex } \times \text { genotype interaction: } F_{(1,47)}=0.183, p=0.833\end{array}$ \\
\hline $\begin{array}{l}\text { Shank3- }(n=24 \\
\text { WT, } 16 \text { HET, } \\
20 \mathrm{KO})\end{array}$ & $\begin{array}{l}\text { Elevated plus } \\
\text { maze }\end{array}$ & Duration in arms & $\begin{array}{l}\text { Sex and genotype, arm } \\
\text { Fig. } 3 M\end{array}$ & $\begin{array}{l}\text { Three-way ANOVA; no main effect of sex: } F_{(1,131)}=0.00005, p=0.994 \text {; } \\
\text { no main effect of genotype: } F_{(2,131)}=0.009, p=0.990 ; \text { no main effect } \\
\text { of arm: } F_{(1,131)}=628.018, p<0.0001 \text {; no main effect of sex } \times \text { geno- } \\
\text { type interaction: } F_{(2,131)}=0.005, p=0.994 ; \text { no main effect of sex } \times \\
\text { arm: } F_{(1,131)}=1.015, p=0.315 ; \text { no main effect of genotype } \times \text { arm: } \\
F_{(1,131)}=0.174, p=0.840 ; \text { no main effect of genotype } \times \text { sex } \times \text { arm: } \\
F_{(2,131)}=1.549, p=0.216\end{array}$ \\
\hline $\begin{array}{l}\text { Shank3+ }(n=24 \\
\text { WT, } 19 \mathrm{HET} \\
16 \mathrm{KO})\end{array}$ & & Duration in arms & $\begin{array}{l}\text { Sex and genotype, arm } \\
\text { Fig. } 3 N\end{array}$ & $\begin{array}{l}\text { Three-way ANOVA; no main effect of sex: } F_{(1,105)}=0.087, p=0.768 \text {; no } \\
\text { main effect of genotype: } F_{(2,105)}=0.314, p=0.730 ; \text { no main effect of } \\
\text { arm: } F_{(1,105)}=708.548, p<0.0001 \text {; no main effect of sex } \times \text { genotype } \\
\text { interaction: } F_{(2,105)}=0.358, p=0.699 ; \text { no main effect of sex } \times \text { arm: } \\
F_{(1,105)}=1.521, p=0.220 ; \text { no main effect of genotype } \times \text { arm: } F_{(1,105)}= \\
2.244, p=0.111 \text {; no main effect of genotype } \times \text { sex } \times \text { arm: } F_{(2,105)}= \\
1.482, p=0.232\end{array}$ \\
\hline $\begin{array}{l}\text { Shank3- }(n=24 \\
\text { WT,16 HET, } 20 \\
\text { KO) }\end{array}$ & Rearing & $\begin{array}{l}\text { Habituation of } \\
\text { rearing }\end{array}$ & $\begin{array}{l}\text { Sex, genotype, bin } \\
\text { Post hoc: Scheffe } \\
\text { Genotype } \\
\text { Fig. } 30\end{array}$ & 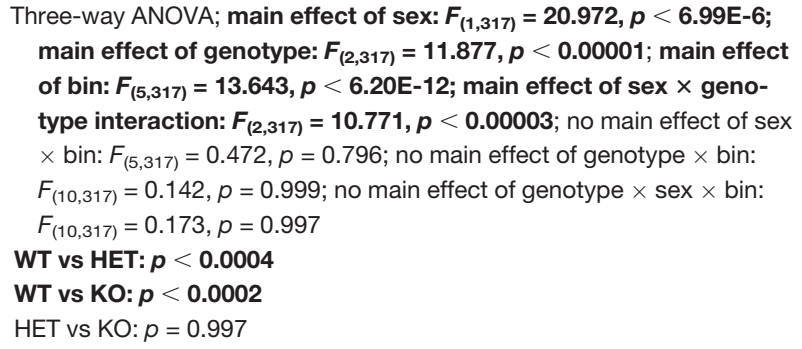 \\
\hline $\begin{array}{l}\text { Shank3+ }(n=24 \\
\text { WT, } 19 \text { HET, } \\
20 \mathrm{KO})\end{array}$ & Rearing & $\begin{array}{l}\text { Habituation of } \\
\text { rearing }\end{array}$ & $\begin{array}{l}\text { Sex, genotype, bin } \\
\text { Fig. } 3 P\end{array}$ & $\begin{array}{l}\text { Three-way ANOVA; no main effect of sex: } F_{(1,255)}=0.342, p=0.322 \text {; no } \\
\text { main effect of genotype: } F_{(2,255)}=0.781, p=0.130 \text {; main effect of } \\
\text { bin: } \boldsymbol{F}_{(\mathbf{2 3 , 2 5 5 )}}=\mathbf{7 8 . 6 7 , p}<\mathbf{0 . 0 0 0 0 1} \text {; no main effect of sex } \times \text { genotype } \\
\text { interaction: } F_{(2,255)}=0.791, p=0.242 ; \text { main effect of sex } \times \text { bin: } \\
\boldsymbol{F}_{(23,255)}=1.39, p<0.0031 \text {; no main effect of genotype } \times \text { bin: } \\
F_{(46,255)}=1.13, p=0.217 \text {; no main effect of genotype } \times \text { sex } \times \\
\text { bin: } F_{(46,255)}=1.223, p=0.0691\end{array}$ \\
\hline $\begin{array}{l}\text { Shank3- }(n=24 \\
\text { WT, } 16 \text { HET, } \\
20 \mathrm{KO})\end{array}$ & Rotarod & Latency to fall & $\begin{array}{l}\text { Sex, genotype, trial } \\
\text { Fig. } 3 S\end{array}$ & 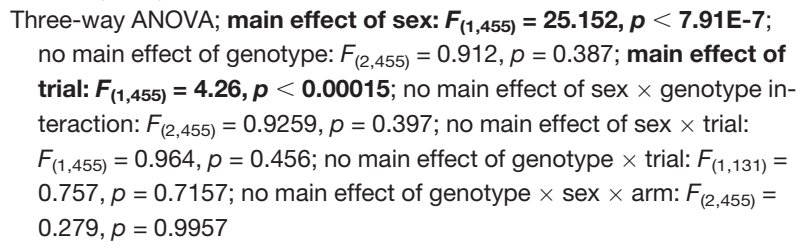 \\
\hline $\begin{array}{l}\text { Shank3+ }(n=24 \\
\text { WT, } 19 \text { HET, } \\
16 \mathrm{KO})\end{array}$ & Rotarod & Latency to fall & $\begin{array}{l}\text { Sex, genotype, trial } \\
\text { Fig. } 3 T\end{array}$ & 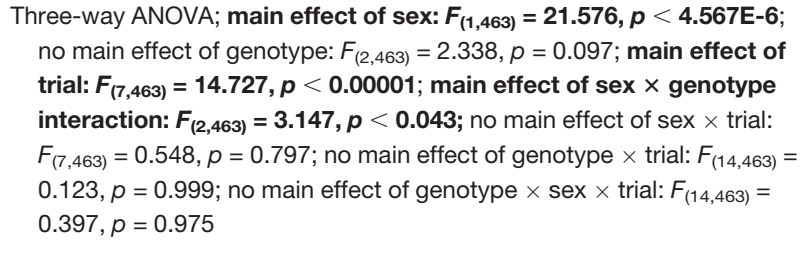 \\
\hline
\end{tabular}


Table 1: Continued

\begin{tabular}{|c|c|c|c|c|}
\hline $\begin{array}{l}\text { Shank3- }(n=24 \\
\text { WT, } 16 \mathrm{HET}, \\
20 \mathrm{KO})\end{array}$ & Locomotor & Beam breaks & $\begin{array}{l}\text { Sex, genotype, bin } \\
\text { Post hoc Scheffe } \\
\text { Genotype } \\
\text { Sex } \\
\text { Female } \\
\text { Male } \\
\text { Fig. } 3 Q\end{array}$ & 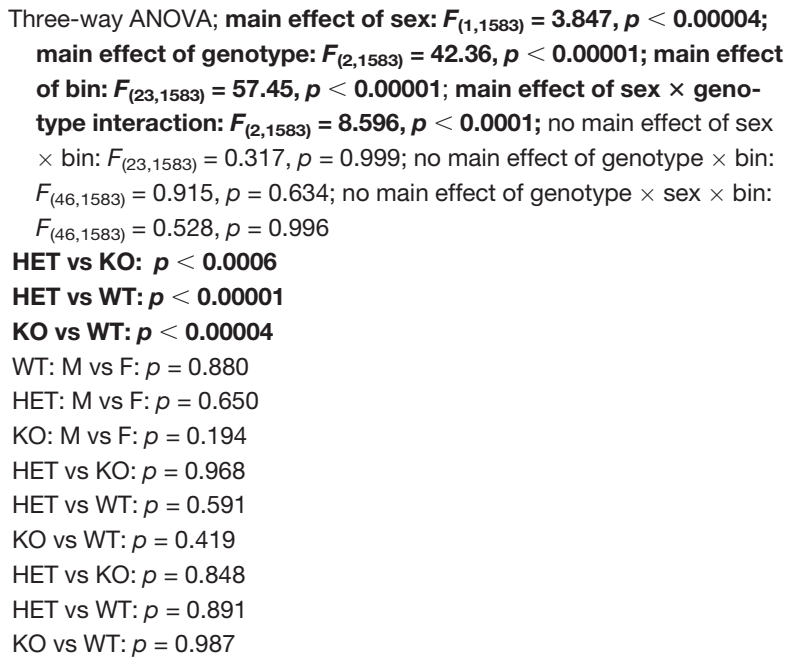 \\
\hline $\begin{array}{c}\text { Shank3+ }(n=24 \\
\text { WT, } 19 \text { HET, } \\
20 \mathrm{KO})\end{array}$ & & Beam breaks & $\begin{array}{l}\text { Sex, genotype, bin } \\
\text { Post hoc Scheffe } \\
\text { Genotype } \\
\text { Sex } \\
\text { male vs female } \\
\text { Female } \\
\text { Male } \\
\text { Fig. } 3 R\end{array}$ & 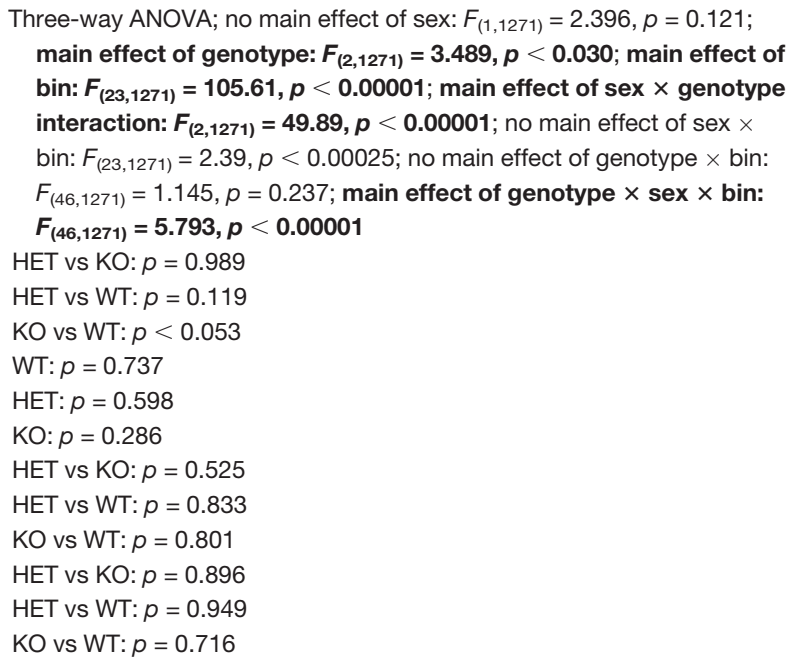 \\
\hline $\begin{array}{l}\text { Shank3- }(n=24 \\
\text { WT, } 16 \text { HET, } \\
20 \mathrm{KO})\end{array}$ & $\begin{array}{l}\text { Morris water } \\
\text { maze }\end{array}$ & $\begin{array}{l}\text { Latency to reach } \\
\text { platform }\end{array}$ & $\begin{array}{l}\text { Sex, genotype, days } \\
\text { Post hoc: Scheffe } \\
\text { Genotype } \\
\text { Sex: female vs male } \\
\text { Female } \\
\text { Male } \\
\text { Fig. } 4 A\end{array}$ & 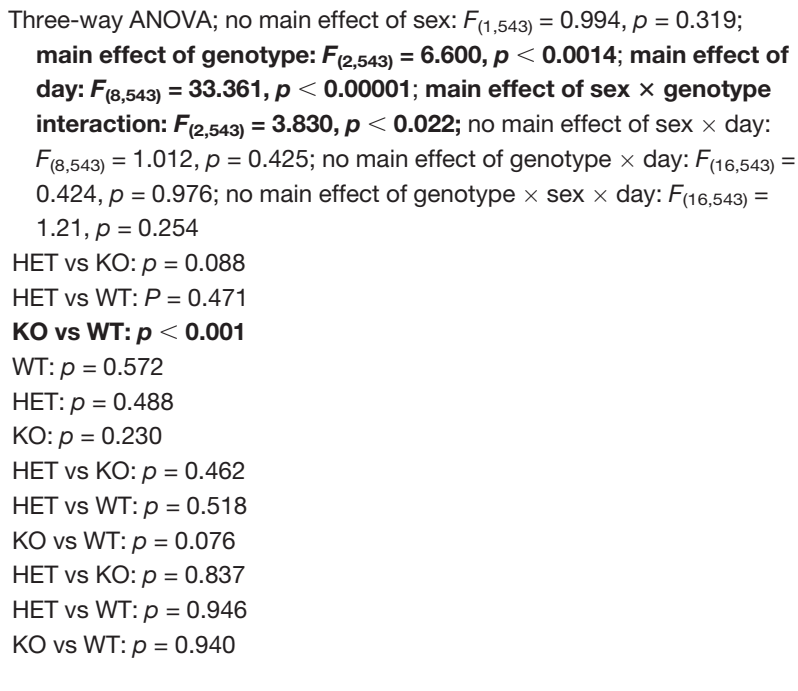 \\
\hline
\end{tabular}




\begin{tabular}{|c|c|c|}
\hline$\%$ Thigmotaxis & $\begin{array}{l}\text { Sex, genotype, days } \\
\text { Post hoc: Scheffe } \\
\text { Genotype } \\
\text { Sex: female vs male } \\
\text { Female } \\
\text { Male } \\
\text { Fig. } 4 B\end{array}$ & 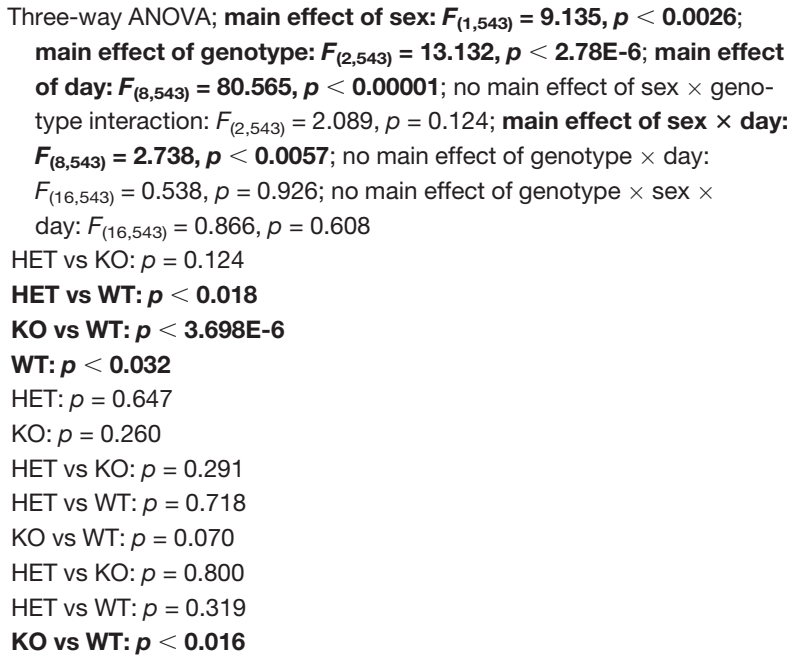 \\
\hline Mean velocity & $\begin{array}{l}\text { Sex, genotype, days } \\
\text { Post hoc: Scheffe } \\
\text { Genotype } \\
\text { Sex: female vs male } \\
\text { Female } \\
\text { KO vs WT: } p<0.013 \\
\text { Fig. } 4 \text { C }\end{array}$ & 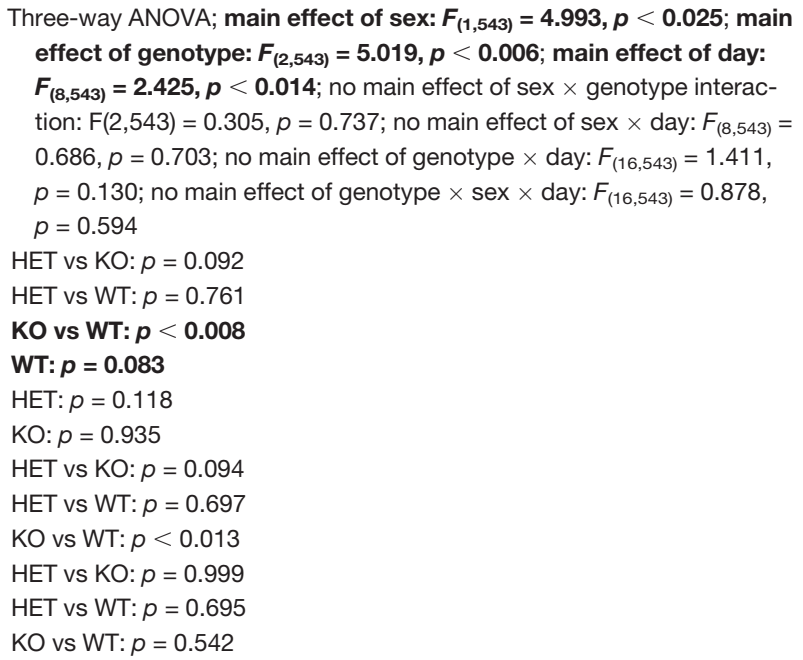 \\
\hline Total distance & $\begin{array}{l}\text { Sex, genotype, days } \\
\text { Post hoc: Scheffe } \\
\text { Genotype } \\
\text { Sex: female vs male } \\
\text { Female } \\
\text { Male } \\
\text { Fig. } 4 D\end{array}$ & 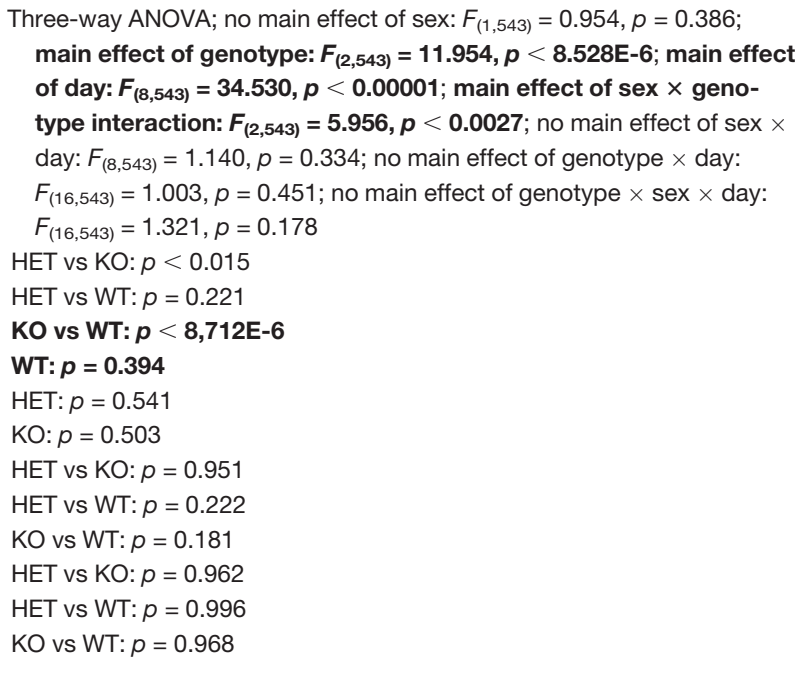 \\
\hline
\end{tabular}




\begin{tabular}{|c|c|c|c|c|}
\hline & Probe trial & $\begin{array}{l}\text { Sex, genotype, location } \\
\text { Post hoc: Scheffe } \\
\text { Location } \\
\text { Fig. } 4 E\end{array}$ & 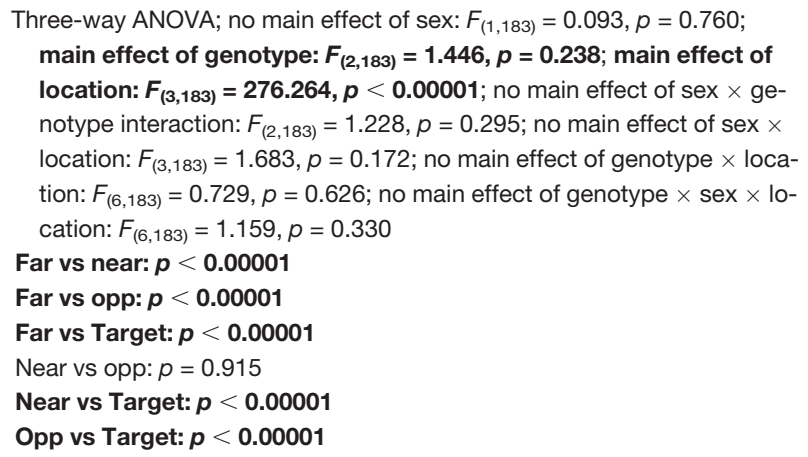 \\
\hline \multirow[t]{4}{*}{$\begin{array}{l}\text { Shank3+ }(n=24 \\
\text { WT, } 19 \text { HET, } \\
20 \mathrm{KO})\end{array}$} & $\begin{array}{l}\text { Morris water } \\
\text { maze }\end{array}$ & $\begin{array}{l}\text { Latency to reach } \\
\text { platform }\end{array}$ & $\begin{array}{l}\text { Sex, genotype, days } \\
\text { Post hoc: Scheffe } \\
\text { Genotype } \\
\text { Fig. } 4 F\end{array}$ & 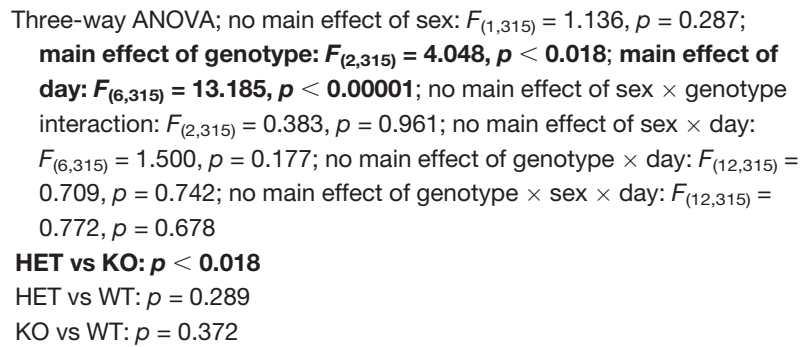 \\
\hline & & $\%$ Thigmotaxis & $\begin{array}{l}\text { Sex, genotype, days } \\
\text { Post hoc: Scheffe } \\
\text { Genotype } \\
\text { Sex: female vs male } \\
\text { Female } \\
\text { Male } \\
\text { Fig. } 4 G\end{array}$ & 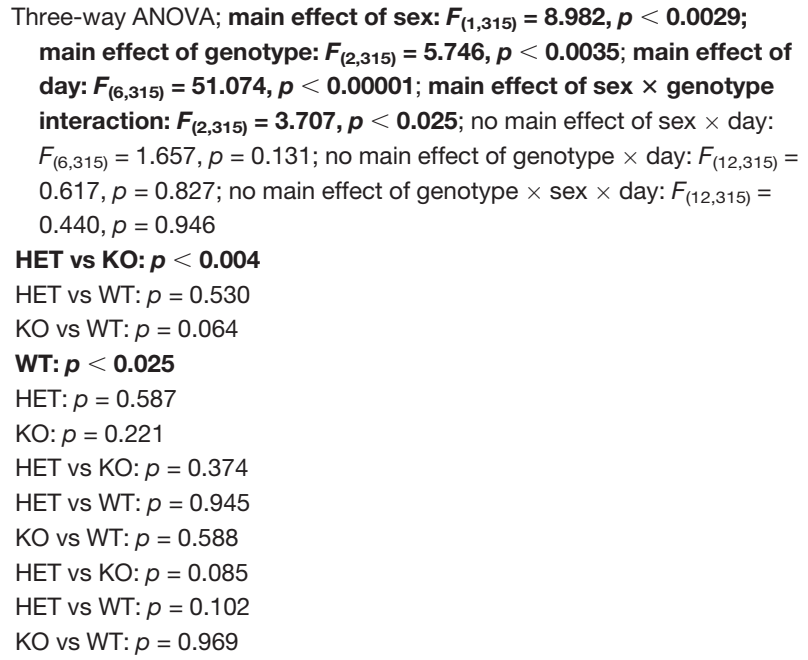 \\
\hline & & Mean velocity & $\begin{array}{l}\text { Sex, genotype, days } \\
\text { Fig. } 4 H\end{array}$ & $\begin{array}{l}\text { Three-way ANOVA; no main effect of sex: } F(1,315)=0.219, p=0.640 \text {; } \\
\text { main effect of genotype: } \boldsymbol{F}_{(2,315)}=0.857, \boldsymbol{p}=0.857 \text {; main effect of } \\
\text { day: } \boldsymbol{F}_{(8,315)}=0.940, \boldsymbol{p}=\mathbf{0 . 4 6 6} \text {, no main effect of sex } \times \text { genotype in- } \\
\text { teraction: } F_{(2,315)}=0.438, p=0.645 ; \text { no main effect of sex } \times \text { day: } \\
F_{(6,543)}=0.763, p=0.763 ; \text { no main effect of genotype } \times \text { day: } F_{(12,315)}= \\
0.922, p=0.524 ; \text { no main effect of genotype } \times \text { sex } \times \text { day: } F_{(12,315)}= \\
1.014, p=0.435\end{array}$ \\
\hline & & Total distance & $\begin{array}{l}\text { Sex, genotype, days } \\
\text { Post hoc: Scheffe } \\
\text { Genotype } \\
\text { Fig. } 4 \text { I }\end{array}$ & $\begin{array}{l}\text { Three-way ANOVA; no main effect of sex: } F_{(1,315)}=2.447, p=0.118 \text {; } \\
\text { main effect of genotype: } \boldsymbol{F}_{(\mathbf{2}, 315)}=\mathbf{3 . 6 7 3 , \boldsymbol { p }}<\mathbf{0 . 0 2 6} \text { main effect of } \\
\text { day: } \boldsymbol{F}_{(6,315)}=10.167, \boldsymbol{p}<\mathbf{3 . 7 1 7 E - 1 0 ;} \text { no main effect of sex } \times \text { geno- } \\
\text { type interaction: } F_{(2,315)}=0.435, p=0.647 ; \text { no main effect of sex } \times \\
\text { day: } F_{(6,315)}=1.527, p=0.169 ; \text { no main effect of genotype } \times \text { day: } \\
F_{(12,315)}=0.853, p=0.594 ; \text { no main effect of genotype } \times \text { sex } \times \text { day: } \\
F_{(12,315)}=0.870, p=0.577 \\
\text { HET vs KO: } \boldsymbol{p}<\mathbf{0 . 0 2 8} \\
\text { HET vs WT: } p=0.227 \\
\text { KO vs WT: } p=0.550\end{array}$ \\
\hline
\end{tabular}




\begin{tabular}{|c|c|c|c|c|}
\hline & & Probe trial & $\begin{array}{l}\text { Sex, genotype, location } \\
\text { Post hoc: Scheffe } \\
\text { Location } \\
\text { Fig. } 4 \mathrm{~J}\end{array}$ & 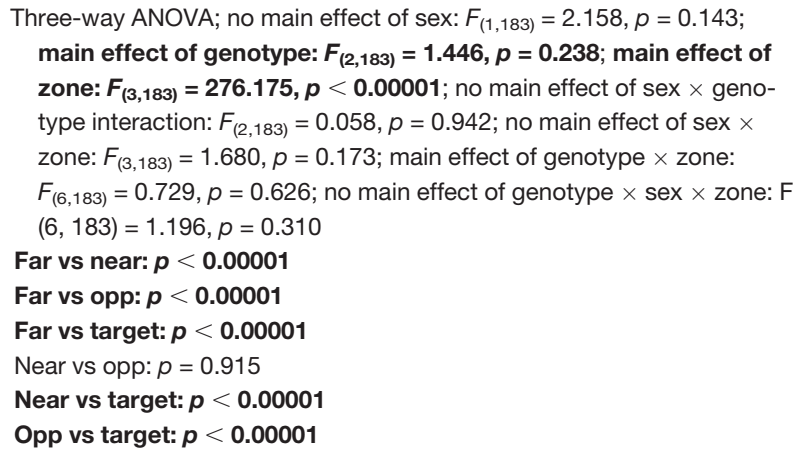 \\
\hline \multirow[t]{2}{*}{$\begin{array}{l}\text { Shank3- }(n=24 \\
\text { WT, } 16 \text { HET, } \\
20 \mathrm{KO})\end{array}$} & Fear conditioning & Cue test & $\begin{array}{l}\text { Genotypes and sex } \\
\text { Fig. } 5 A\end{array}$ & $\begin{array}{l}\text { Two-way ANOVA; no main effect of sex: } F_{(1,52)}=2.774, p=0.102 ; \text { no } \\
\text { main effect of genotype: } F_{(2,52)}=1.991, p=0.147 ; \text { no main effect of } \\
\text { sex } \times \text { genotype interaction: } F_{(1,52)}=0.669, p=0.516\end{array}$ \\
\hline & & Contextual test & $\begin{array}{l}\text { Genotypes and sex } \\
\text { Fig. } 5 B\end{array}$ & $\begin{array}{l}\text { Two-way ANOVA; no main effect of sex: } F_{(1,64)}=1.426, p=0.237 \text {; no } \\
\text { main effect of genotype: } F_{(2,64)}=0.423, p=0.656 \text {; no main effect of } \\
\text { sex } \times \text { genotype interaction: } F_{(1,64)}=1.347, p=0.2677\end{array}$ \\
\hline \multirow[t]{2}{*}{$\begin{array}{l}\text { Shank3+ }(n=24 \\
\text { WT, } 19 \text { HET, } \\
20 \mathrm{KO})\end{array}$} & Fear conditioning & Cue test & $\begin{array}{l}\text { Genotypes and sex } \\
\text { Fig. } 5 C\end{array}$ & $\begin{array}{l}\text { Two-way ANOVA; no main effect of sex: } F_{(1,52)}=2.774, p=0.102 \text {; no } \\
\text { main effect of genotype: } F_{(2,52)}=1.991, p=0.147 ; \text { no main effect of } \\
\text { sex } \times \text { genotype interaction: } F_{(1,52)}=0.669, p=0.516\end{array}$ \\
\hline & & Contextual test & $\begin{array}{l}\text { Genotypes and sex } \\
\text { Fig. } 5 D\end{array}$ & $\begin{array}{l}\text { Two-way ANOVA; no main effect of sex: } F_{(1,52)}=0.0007, p=0.978 \text {; no } \\
\text { main effect of genotype: } F_{(2,52)}=1.586, p=0.215 ; \text { no main effect of } \\
\text { sex } \times \text { genotype interaction: } F_{(1,52)}=2.491, p=0.0936\end{array}$ \\
\hline \multirow[t]{2}{*}{$\begin{array}{l}\text { Shank3- }(n=24 \\
\text { WT, } 16 \text { HET, } \\
20 \mathrm{KO})\end{array}$} & Nesting & Width & $\begin{array}{l}\text { Genotypes, sex, time } \\
\text { Post hoc: Scheffe } \\
\text { Genotype } \\
\text { Time } \\
\text { Fig. } 5 F\end{array}$ & 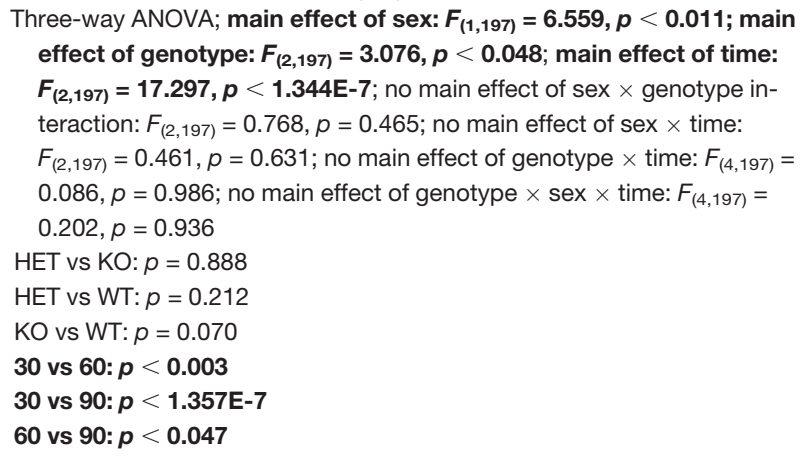 \\
\hline & & Height & $\begin{array}{l}\text { Genotypes, sex, time } \\
\text { Post hoc: Scheffe } \\
\text { Genotype } \\
\text { Fig. } 5 E\end{array}$ & 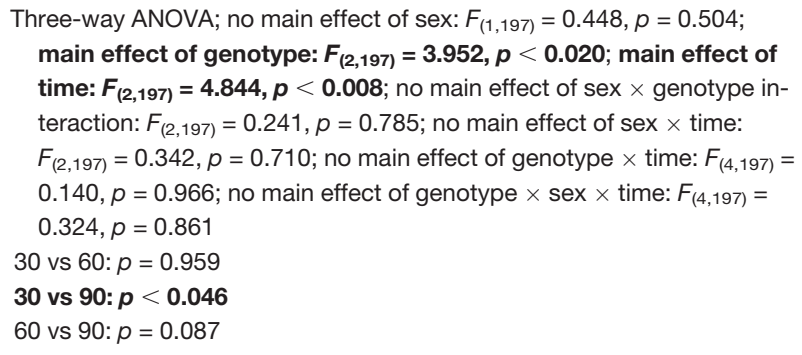 \\
\hline $\begin{array}{l}\text { Shank3+ }(n=24 \\
\text { WT, } 19 \text { HET, } \\
20 \mathrm{KO})\end{array}$ & Nesting & Width & $\begin{array}{l}\text { Genotypes, sex, time } \\
\text { Post hoc: Scheffe } \\
\text { Time } \\
\text { Fig. } 5 H\end{array}$ & 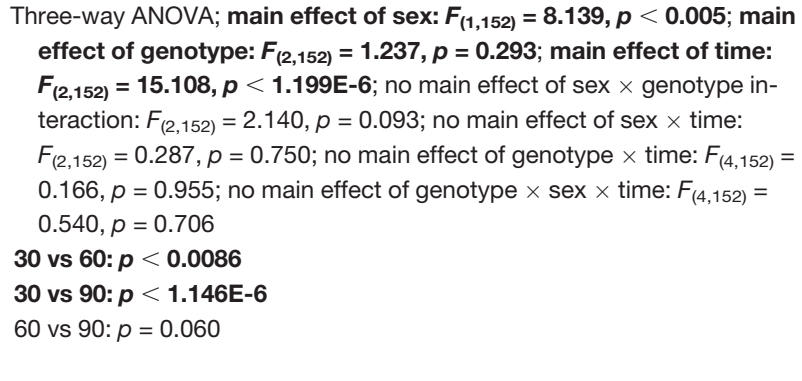 \\
\hline
\end{tabular}


Table 1: Continued

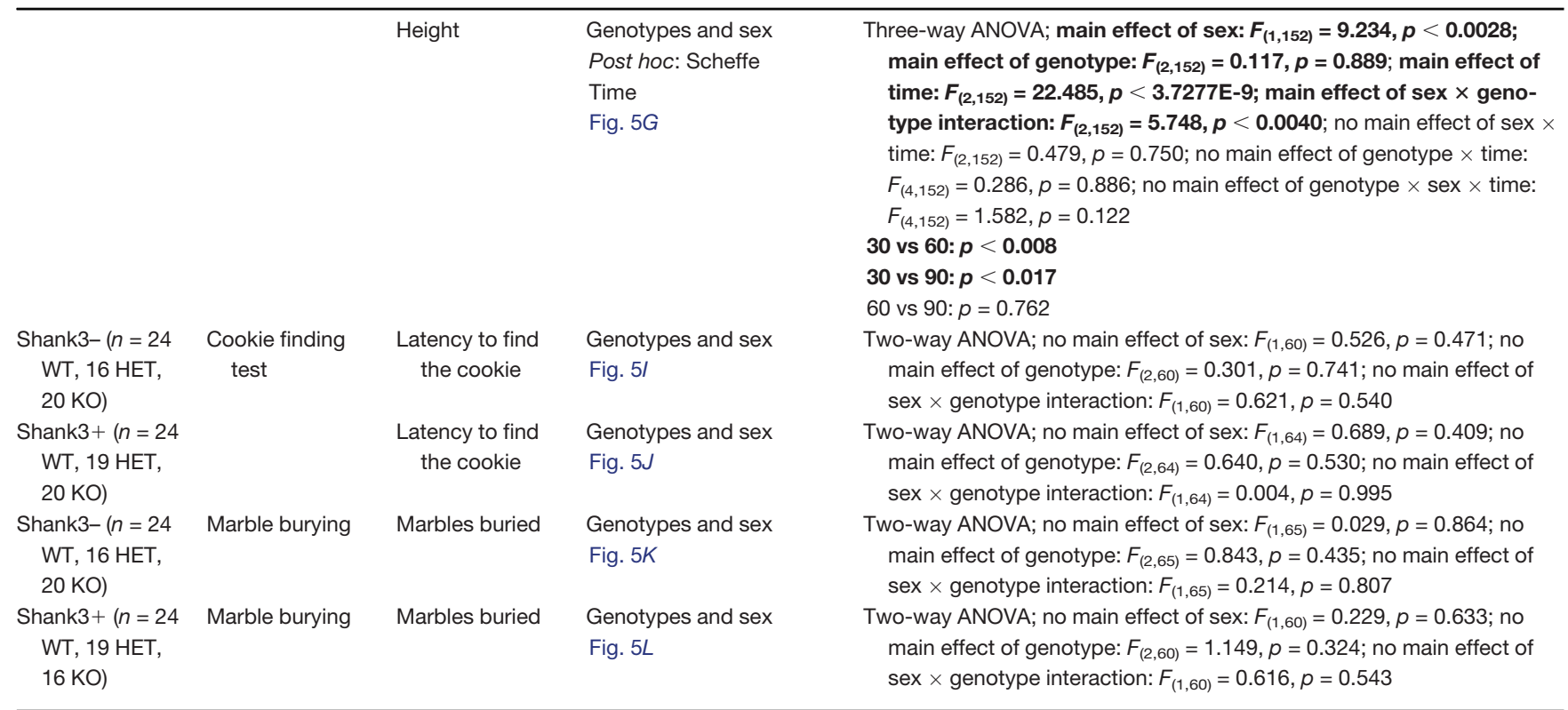

revolution it was scored as a fall. Each mouse received four trials per day for $2 \mathrm{~d}$. Within a day, each trial had an intertrial interval of $\sim 45 \mathrm{~min}$.

$\mathrm{OF}$

The OF test was conducted as described previously (Etherton et al., 2009). Briefly, mice were placed along the edge of an open arena ( $44 \times 44 \times 44 \mathrm{~cm}, \sim 7$ lux $)$ and allowed to freely explore for $10 \mathrm{~min}$. Mice were monitored using CleverSys TopScan Software.

\section{Social interaction tests}

Social interaction with a novel juvenile target mouse was performed essentially as described (Kwon et al., 2006; Tabuchi et al., 2007; Blundell et al., 2009). Briefly, following a 15-min habituation under red light, the experimental and target mice (novel BALB/cByJ juvenile mouse; three weeks of age; The Jackson Laboratory, stock \#001026) were placed in a novel cage for 2 min and allowed to directly interact. Interaction was scored by observing the duration and number of times the test mouseinitiated contact with or sniffed the juvenile mouse. Contact was considered as any part of the body touching the juvenile mouse. Three days later, the same experimental and juvenile mice were paired again in a novel cage for 2 min and scored in the same manner. Social interaction with a caged adult was performed as described (Blundell et al., 2010). Briefly, the test was performed in a $48 \times 48 \mathrm{~cm}^{2}$ white plastic arena under red light using 3.5 " $\times 2$ " $\times 30$ " clear rectangular cage containing a BALB/ cByJ novel adult mouse. The lower half of the rectangular cage has small openings to allow for olfactory and minimal tactile interaction. Initially mice were allowed to explore the arena for 5 min with an empty rectangular cage. Then mice were allowed to approach a novel adult mouse housed in the rectangular cage for another $5 \mathrm{~min}$. Social interaction with genotype-matched and sex-matched pairs was performed by pairing mice with a sex-matched and genotypematched partner within the experimental cohort. Matched pairs were derived from separate cages and were never previously housed together. Mouse pairs were placed at separate ends in an OF arena $(44 \times 44 \times 44 \mathrm{~cm})$ and allowed to interact for $5 \mathrm{~min}$ under dim lighting ( $\sim 7$ lux). For threechamber social approach, social preference and social novelty were tested using a three-chambered box as described previously (Blundell et al., 2009) and based to a large extent on the original descriptions (Moy et al., 2004; Nadler et al., 2004). This test consisted of three, 10-min trials. During the first trial, the mouse was allowed to explore the three-chamber box in which each end-chamber contained an empty cage (upside down pencil holder). In the second trial, the three-chamber box contained a novel BALB/cByJ stimulus mouse under a cage in one of the end-chambers and an empty cage in the opposite end-chamber. The test mouse was free to choose between an inanimate cage and a caged social target. For the third trial, the test mouse was free to choose between a caged novel social target (novel BALB/ cByJ mouse) versus the same caged mouse in trial 2 (familiar social target). Locations of empty cages and social targets were counterbalanced, and mice were placed back into the home cage for very brief intervals between trials. Social interactions were objectively monitored and scored using video-tracking and automated CleverSys SocialScan Software.

\section{Grooming}

Mice were placed into an empty cage and allowed to habituate for $10 \mathrm{~min}$ in a room with $\sim 40$ lux of white light. At the end of $10 \mathrm{~min}$, the mice were monitored and video recorded for $10 \mathrm{~min}$ for later analysis. The number of 
A

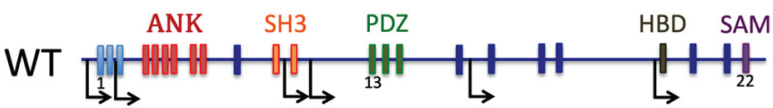

Neo-Stop

Insertion

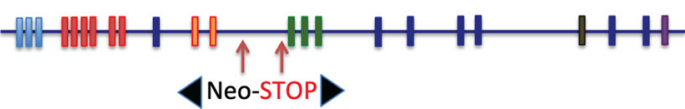

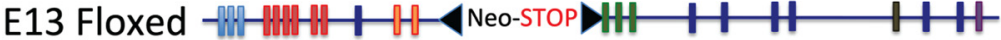

B

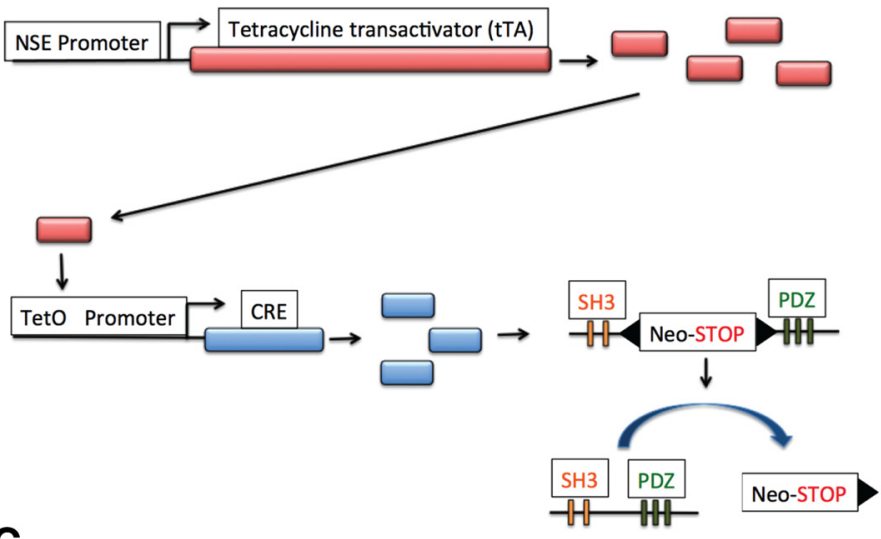

C
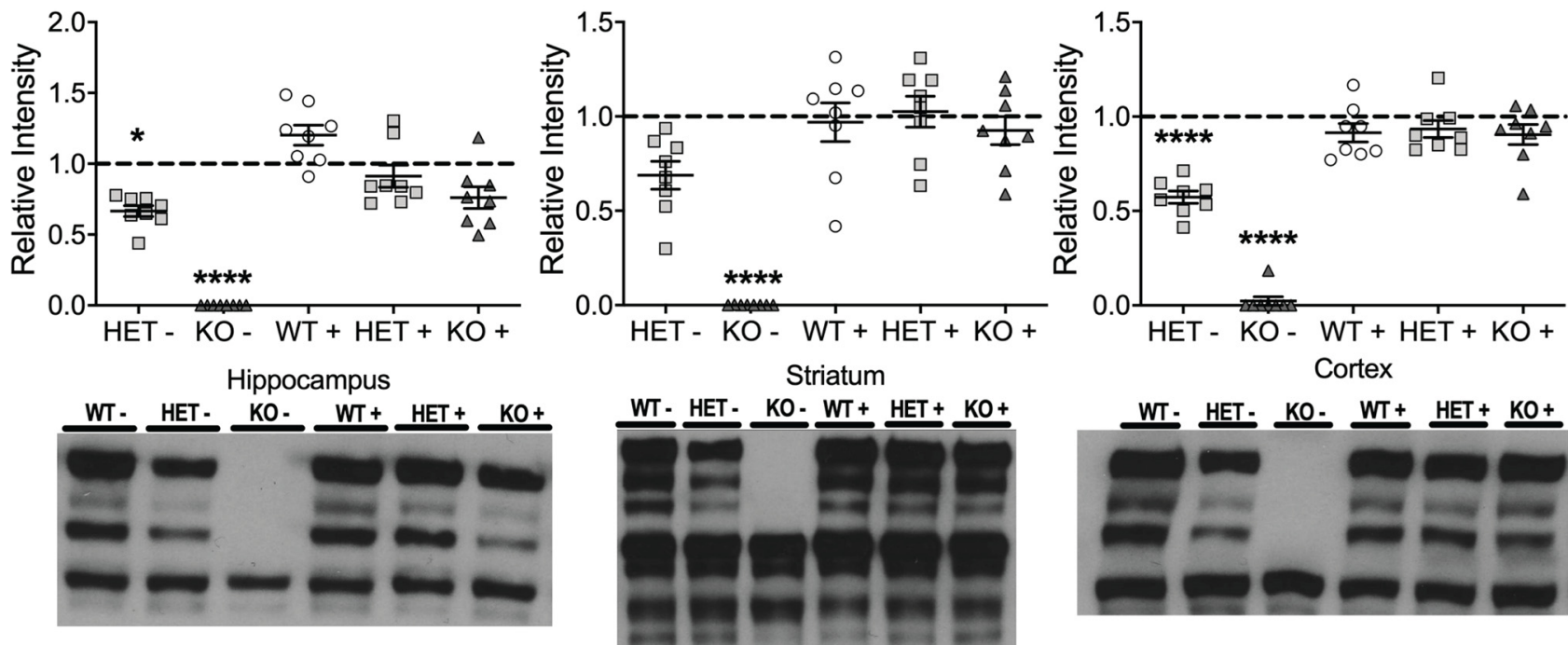

Figure 1. Generation of genetically reversible Shank3 mutant mice. A, top, A diagram of Shank3 gene along with its 22 exons (colored bars), six known promoters (arrows), and its five conserved domains, Ankyrin repeat domain (ANK), Src homology 3 (SH3) domain, PDZ domain, a proline-rich region containing homer- and cortactin-binding sites (HBD), and a sterile $\alpha$ motif (SAM) domain. Middle, Insertion of the neo-stop cassette flanked by loxP sites (black arrowheads) into intron 12 just before exon 13 of the Shank3 gene. Bottom, The generation of the Shank3 ${ }^{\text {FloxE13 }}$ following insertion of the neo-stop cassette. $\boldsymbol{B}$, Regulation of Shank3 gene expression. Top, Schematic representation of the NSE-tTA transgene. Following NSE promoter activation, tTA expression binds the tetO promoter and thus drives Cre expression. Bottom, Cre protein binds to the loxP sites flanking the regulatory cassette and, following recombination, excision of the neo-STOP results in restoration of WT Shank3 gene sequence. $\boldsymbol{C}$, Biochemical analysis in whole lysate preps from hippocampus, striatum, and cortical tissue from both genetically reversed Shank3 ${ }^{\mathrm{E} 13}$ mice $(\mathrm{WT}+$, HET+, $\mathrm{KO}+$ ) and Shank3 ${ }^{\mathrm{E} 13}$ control mice (HET- and KO-; WT- is represented by the dash line); $* p<0.05 ; * * * * p<0.0001 ; n=8 \mathrm{WT}-, n=$ $8 \mathrm{HET}-, n=8 \mathrm{KO}-, n=8 \mathrm{WT}+, n=8 \mathrm{HET}+, n=8 \mathrm{KO}+$. 


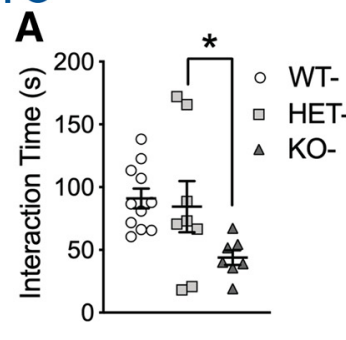

E

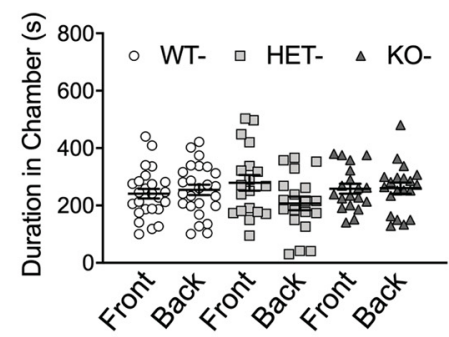

H

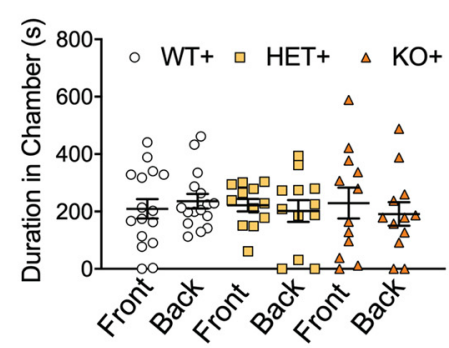

B

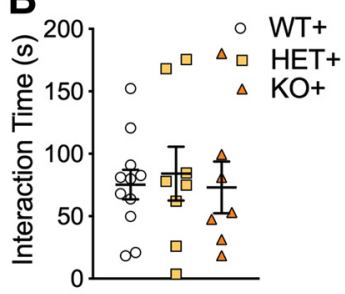

C

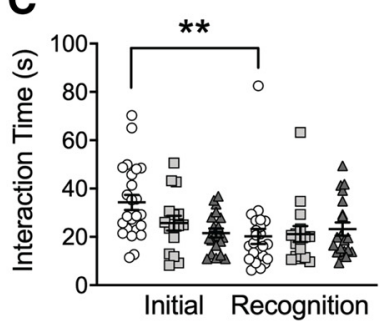

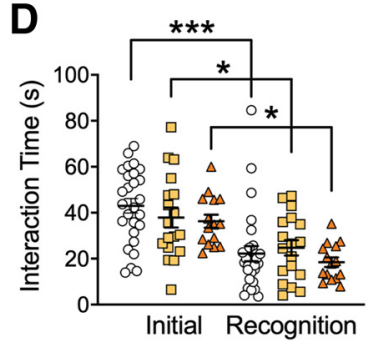

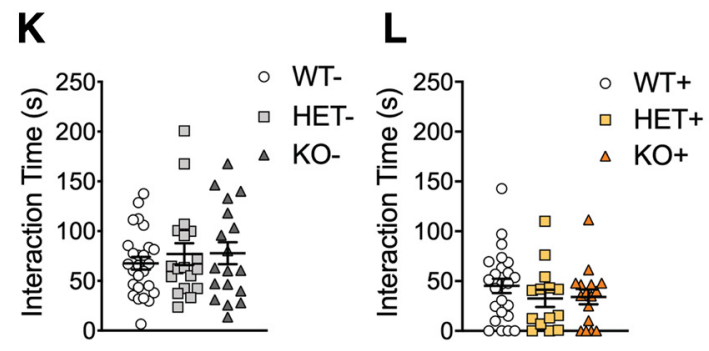

Figure 2. Rescue of social interaction deficits in Shank3+ mutant mice. In the genotype/sex-matched social interaction test, Shank3 KOmice displayed a significant reduction in social interaction time $(\boldsymbol{A})$ compared with HET- mice. Shank3+ mice, including both genetic reversal transgenes, all displayed similar durations of social interaction $(\boldsymbol{B})$ in the genotype/sex-matched social interaction test. In the social interaction with juvenile test of social memory, both HET and $\mathrm{KO}$ mice display deficits in social recognition (C). In the genetically reversed Shank3+ mice, however, all genotypes displayed similar levels of social memory $(\boldsymbol{D})$. In the three-chamber test of sociability, Shank3- mice did not display a chamber preference in the initial trial $(\boldsymbol{E})$. In second trial of this task, Shank3 HET- mice did not display a preference for the social target over the inanimate object $(\boldsymbol{F})$. In the third trial of this task, neither Shank3 HET- nor KO- mice displayed a preference for social novelty $(\boldsymbol{G})$. Shank3+ mice also did not display a baseline chamber preference in the initial trial of the three-chamber test $(\boldsymbol{H})$. In the second trial, Shank3 HET + mice did not display a preference for the social target over the inanimate object (I). In the third trial of this task, Shank3 $\mathrm{HET}+$ mice did not display a preference for social novelty $(\boldsymbol{J})$. Shank3- mice $(\boldsymbol{K})$ along with Shank3+ mice $(\boldsymbol{L})$ both display similar social interaction times, respectively, in approach of a novel, caged social target in the caged conspecific test. Data represented as mean \pm SEM; $* p<0.05 ; * * p<0.01, * * * p<0.001$, ***** $<0.0001$; genotype sex match test $n=11 \mathrm{WT}-, n=8 \mathrm{HET}-, n=7 \mathrm{KO}-; n=11 \mathrm{WT}+, n=8$ $\mathrm{HET}+, n=7 \mathrm{KO}+$; all other behaviors $n=24 \mathrm{WT}-, n=16 \mathrm{HET}-, n=20 \mathrm{KO}-, n=24 \mathrm{WT}+, n=19 \mathrm{HET}+, n=16 \mathrm{KO}+$.

grooming bouts along with the total time spent grooming was measured. Time spent grooming the face, head, body, or tail are all considered grooming.

\section{Morris water maze}

The Morris water maze task was conducted as previously described (Powell et al., 2004). Briefly, in a 120-cm diameter pool, a $10-\mathrm{cm}$ diameter circular platform was submerged $\sim 1 \mathrm{~cm}$ below the surface of the water $\left(22 \pm 1^{\circ} \mathrm{C}\right)$ made opaque with white, non-toxic tempera paint. After finding the platform or being guided by the experimenter to the platform if the 60-s trial limit elapsed, mice remained on the platform for $15 \mathrm{~s}$ before being removed and returned to their home cage. Training was conducted over nine consecutive days, 
A

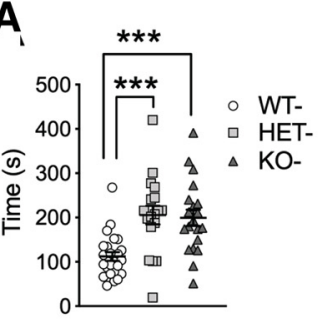

$\mathbf{F}$

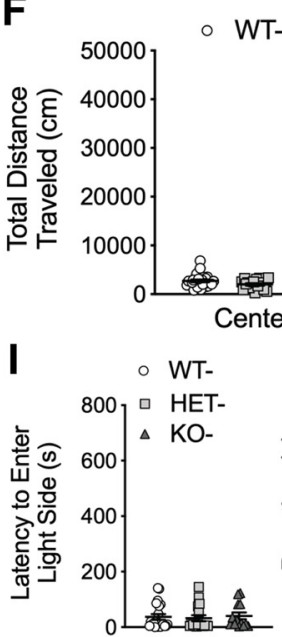

B

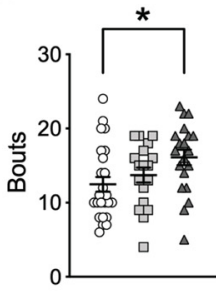

C

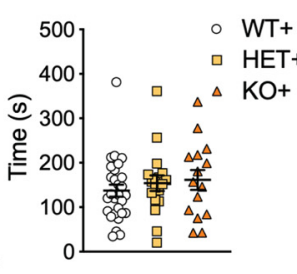

G

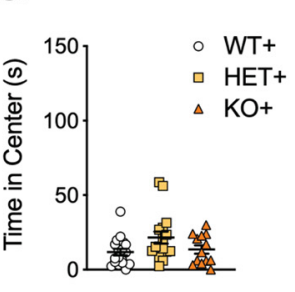

K
E

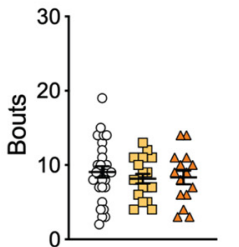

H
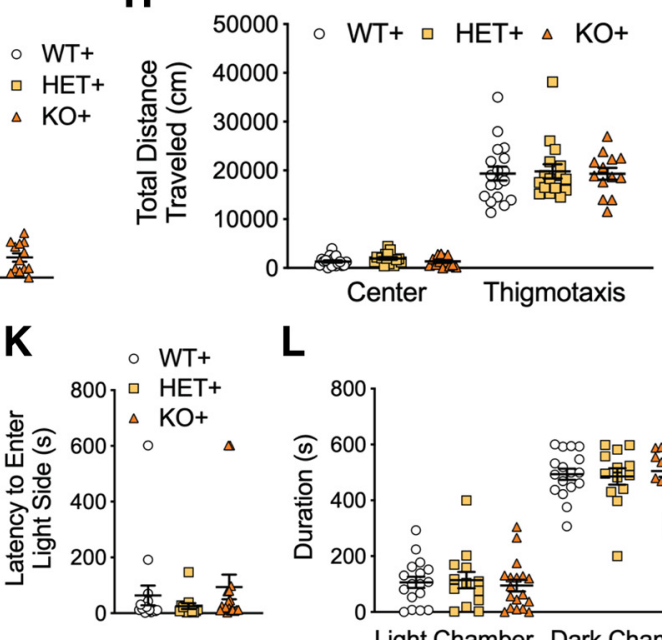

$\mathbf{L}$
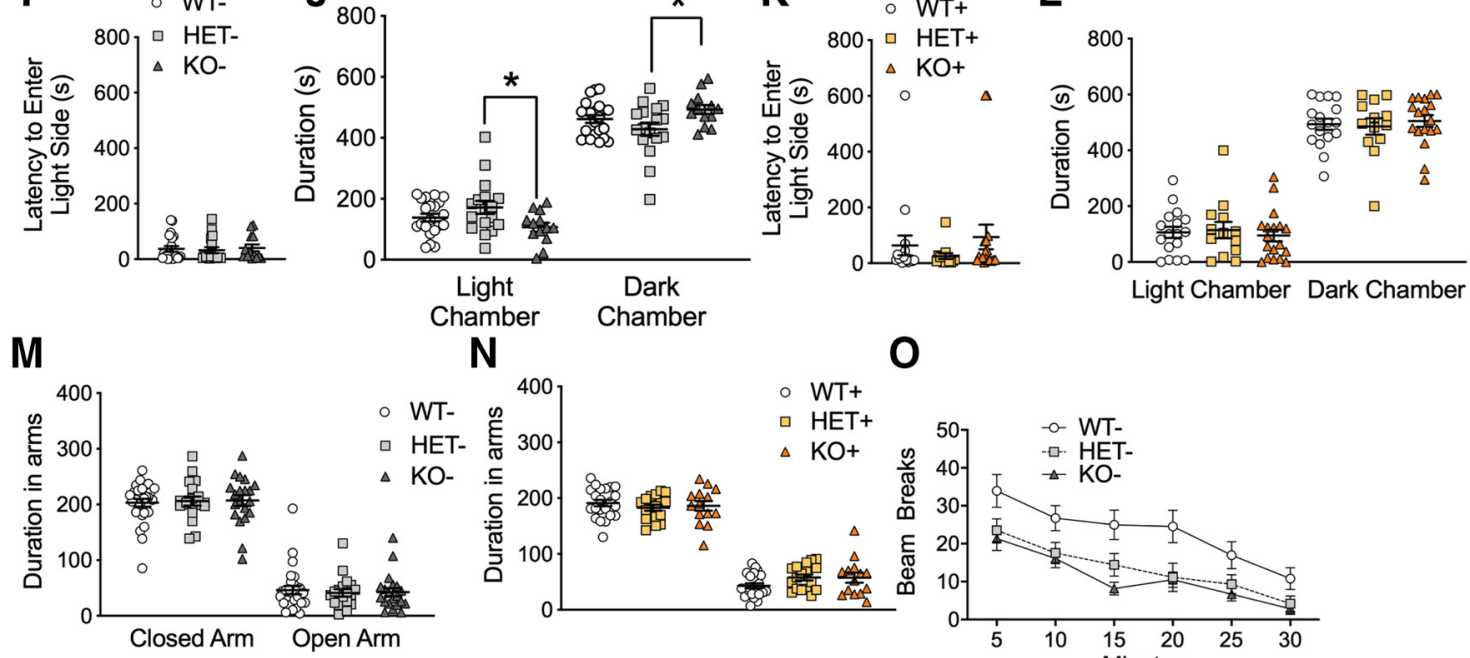

$\mathbf{P}$

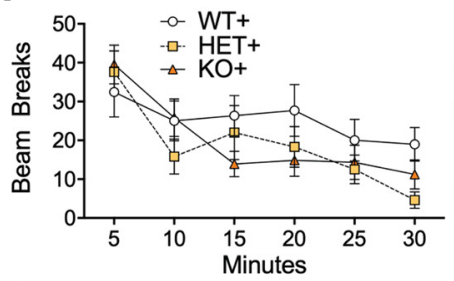

Q

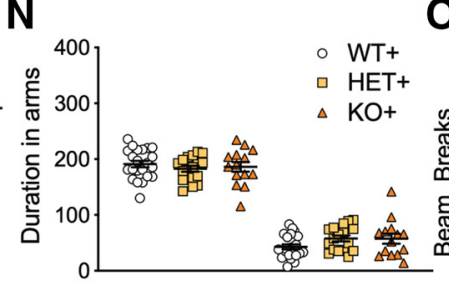

0
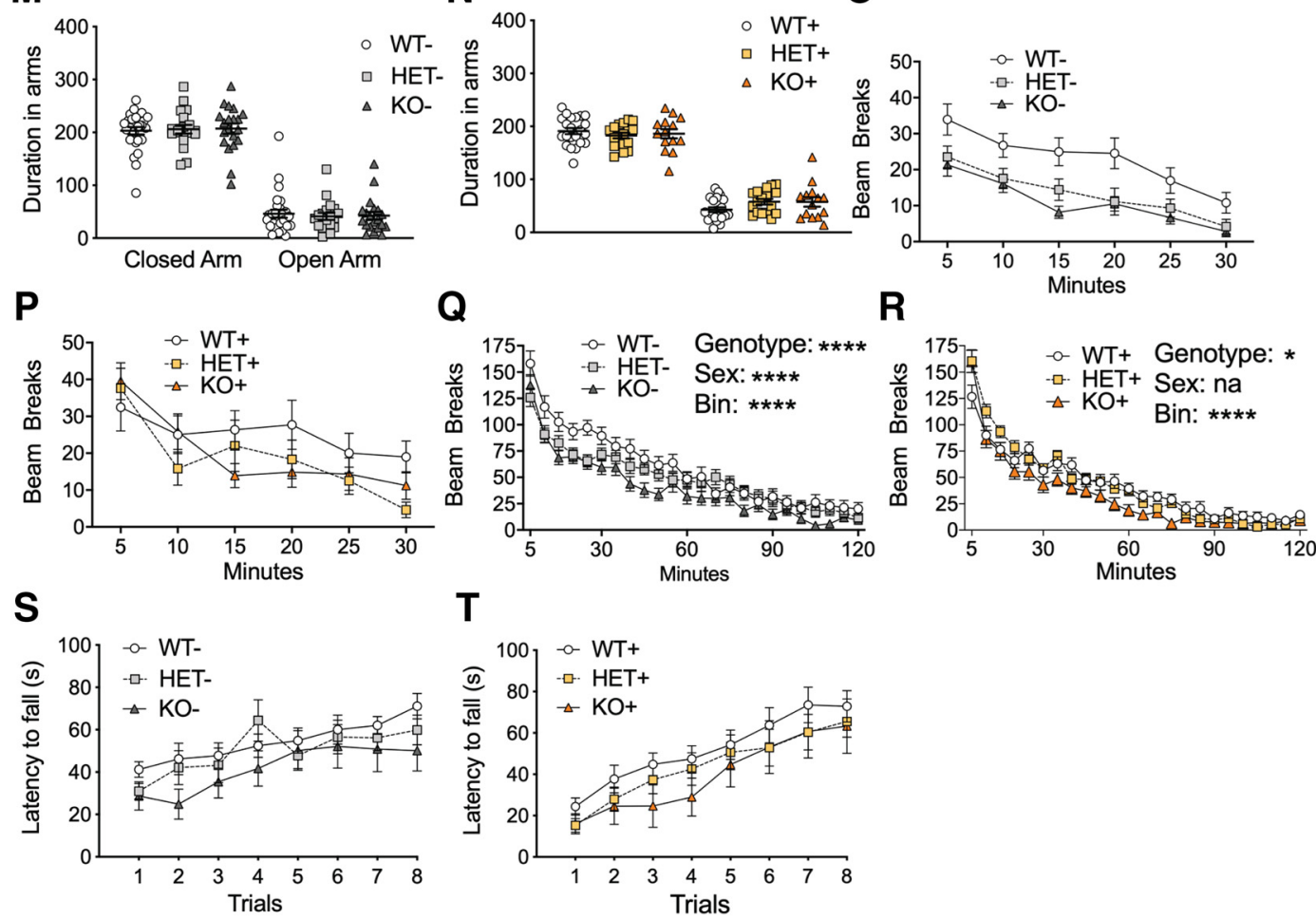

T

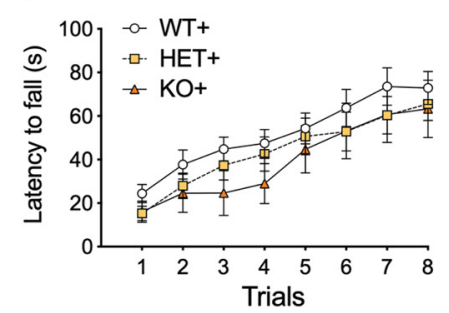

Figure 3. Rescue of increase repetitive grooming in Shank3+ mutant mice. $\boldsymbol{A}$, Shank3 HET- and KO- mice displayed a significant increase in time spent grooming compared with WT- littermate mice. $\boldsymbol{B}$, KO- mice also display a significant increase in the number of grooming bouts compared with WT mice. C, Genetically rescued Shank3 WT+, HET+, and KO+ mice all displayed similar grooming duration and $(\boldsymbol{D})$ number of grooming bouts. In the OF test, Shank3- mice all displayed similar duration in the center (E). Additionally, Shank3- mice displayed similar distances traveled in the center and thigmotaxis regions, respectively $(\boldsymbol{F})$. Shank3+ mice displayed similar duration in the center of the OF $(\mathbf{G})$ and similar distances traveled in the center and thigmotaxis regions $(\boldsymbol{H})$. 
continued

In the light dark test, Shank3- mice displayed similar latencies to enter the light side (I). Shank3 HET- mice displayed increase duration in the light chamber compared with KO- mice $(\boldsymbol{J})$. Additionally, HET- mice displayed a decrease in time spent in the dark chamber compared with KO- mice $(\boldsymbol{J})$. None of the Shank3+ mice displayed a significant difference in their latency to enter the light side $(\boldsymbol{K})$. Also, Shank3+ mice displayed similar duration in light chamber and dark chamber, respectively ( $\boldsymbol{L})$. In the elevated plus maze Shank3- mice displayed similar durations in the closed arm and open arm, respectively (M). Shank3+ mice displayed similar duration in closed arms and open arms, respectively $(\boldsymbol{N})$. In the rearing test Shank3 HET- and KO- mice displayed reduced rearing compared with WT- mice $(\boldsymbol{O})$. Shank3 $\mathrm{HET}+$ and $\mathrm{KO}+$ mice displayed similar rearing behavior when compared with WT + mice $(\boldsymbol{P})$. In the locomotor test KO- mice displayed a significant reduction in beam breaks over a 2-h time period when compared with HETand WT- mice (Q). Shank3 WT+, HET+ and KO+ mice all displayed similar levels of beam breaks $(\boldsymbol{R})$. Both Shank3- (S) and Shank3+ $(\boldsymbol{T})$ mice displayed similar latencies to fall of the rotarod, respectively. Data represented as mean $\pm \mathrm{SEM} * \mathrm{C}<0.05$; $* * p<0.01$, ***p $<0.001$, **** $p<0.0001, n=24 \mathrm{WT}-, n=16 \mathrm{HET}-, n=20 \mathrm{KO}-, n=24 \mathrm{WT}+, n=19 \mathrm{HET}+, n=16 \mathrm{KO}+$.

followed by a probe trail on day 10 (60-s swim with no platform). To test basic visual function, we measured the latency to reach the platform with a visible cue atop the platform in the water maze on day 10.

\section{Marble burying}

As described previously (Blundell et al., 2010b), individual mice were placed in a novel home cage with $5 \mathrm{~cm}$ of bedding. 20 black marbles (16 $\mathrm{mm}$ in diameter) were evenly placed on top of the bedding throughout the home cage, and mice were free to explore the cage for $30 \mathrm{~min}$. After $30 \mathrm{~min}$, the number of marbles buried was recorded. A marble was defined as buried when $<25 \%$ of the marble was visible. This test was conducted in a dimly lit room ( $~ 80$ lux).

\section{Fear conditioning}

Fear conditioning was conducted as previously described (Powell et al., 2004). Mice were placed into a shock box with clear front and rear walls (medAssociates). After a 2-min exploration period, three $30-\mathrm{s}, 90-\mathrm{dB}$ acoustic conditioned stimuli (white noise) followed by a 2-s, 0.5-mA foot shock with 2-min interstimulus intervals were delivered. Mice remained in the chamber 2 min after the last conditioned stimulus/foot shock pairing. Freezing behavior (motionless except respirations) was monitored at 5-s intervals by an observer blind to the genotype. To test 24-h contextual memory, mice were placed into the same training box for $5 \mathrm{~min}$ and scored for freezing behavior every $5 \mathrm{~s}$. Four hours following contextual memory testing, cue-dependent fear conditioning was tested. Mice were placed in a novel environment supplemented with vanilla odor for a 3-min baseline followed by 3 min of conditioned stimulus (tone). Freezing behavior was scored as contextual fear conditioning.

\section{Nesting behavior}

Mice were placed into a novel empty cage with a $5 \times 5 \mathrm{~cm}$ square of pressed cotton (Nestlet; Ancare). The net increase in nest width and height were measured after 30,60 , and $90 \mathrm{~min}$.

\section{Olfactory cookie test}

Mice were not habituated to the cookie before testing. Half of a cookie (Nutter Butter, Nabisco) was buried $\sim 1 \mathrm{~cm}$ under mouse bedding in a novel, clean mouse cage. A test mouse was placed in the cage, and the latency to find the cookie was recorded.

\section{Statistics}

Statistical analyses of behavioral data were conducted using StatPlus software (version 6, AnalystSoft) using either two-way ANOVAs or three-way repeated measure, where applicable. Post hoc Scheffe contrast of means was applied for significant effects and interactions. Figures are represented as mean \pm SEM.

\section{Results}

\section{Generation of Shank3 mutant mice}

In our previous study, we successfully targeted disruption of the Shank3 gene in mice by insertion of a transcriptional neostop cassette containing flanking loxP sites into intron 12, which we term Shank3 ${ }^{\mathrm{E} 13}$ (Fig. 1A). We then created two separate lines of mice by crossing mice heterozygous for Shank ${ }^{\mathrm{E} 13}$ with a NSE-tTA transgene mouse line (gift from Eric Nestler) or Tg(tetO-Cre)1Jaw/J transgene mouse line (The Jackson Laboratory, stock \#006224), to generate

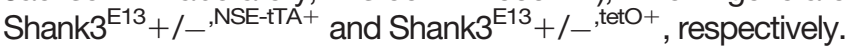
These mice were then bred together to generate mice positive for both transgenes, in addition to being WT (Shank3 ${ }^{\mathrm{E} 13}-/$ ${ }^{,{ }^{\text {tg }}{ }^{++}}$; WT + ), heterozygous (Shank $3^{\mathrm{E} 13}+/{ }^{,{ }^{, t++}+}$; $\mathrm{HET}+$ ), or homozygous (Shank3 $3^{\mathrm{E} 13+/+, t g++;} \mathrm{KO}+$ ), for the floxed neostop cassette upstream of exon 13 of Shank3. The presence of both transgenes allowed for a 2-transgene regulatory system that can remove the floxed neo-stop cassette and thus restore the WT Shank3 gene. In this 2-transgene regulatory system, NSE-tTA activation allows for the expression of tTA (tetracycline transactivator), which binds to the tetO (tetracycline-responsive promoter element) promoter that activates expression of cre-recombinase. Cre will bind to the loxP sites (Fig. 1, black triangles) flanking the neo-stop cassette and allow for removal of the cassette following recombination (Fig. 1B). Whole-lysates from the hippocampus, striatum, and cortex showed that control mice lacking one of the two transgenes (HET- and KO-) displayed reduced SHANK3 expression in all three brain regions. Mice containing both transgenes (WT+, HET+, and $\mathrm{KO}+$ ), however, all displayed similar levels of SHANK3 expression compared with WTmice (Fig. $1 C$, dashed line), thereby demonstrating complete rescue at the level of protein expression.

\section{Lack of brain region selectivity and expected doxycycline regulation}

We initially chose this combination of NSE-tTA and TetO-cre transgenes for dual control purposes. First, 

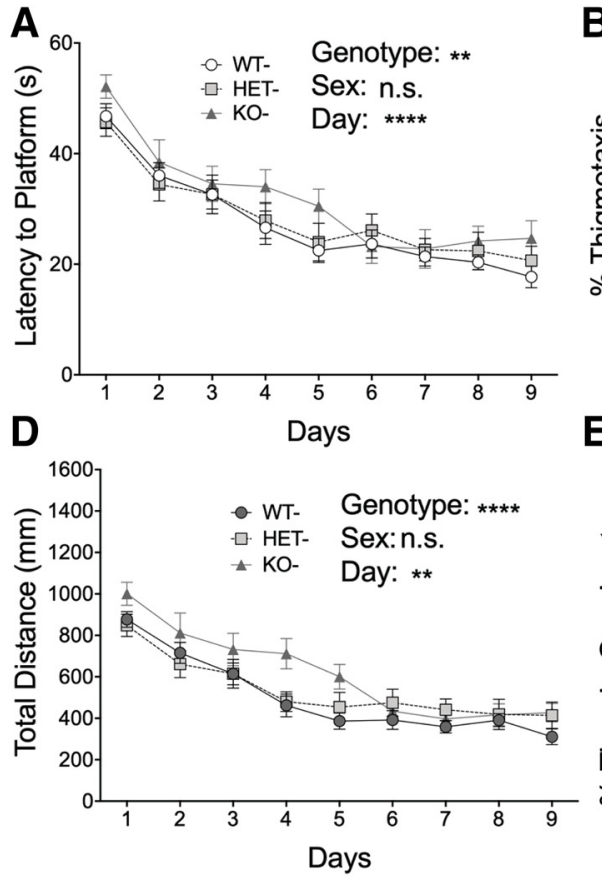

$\mathbf{F}$
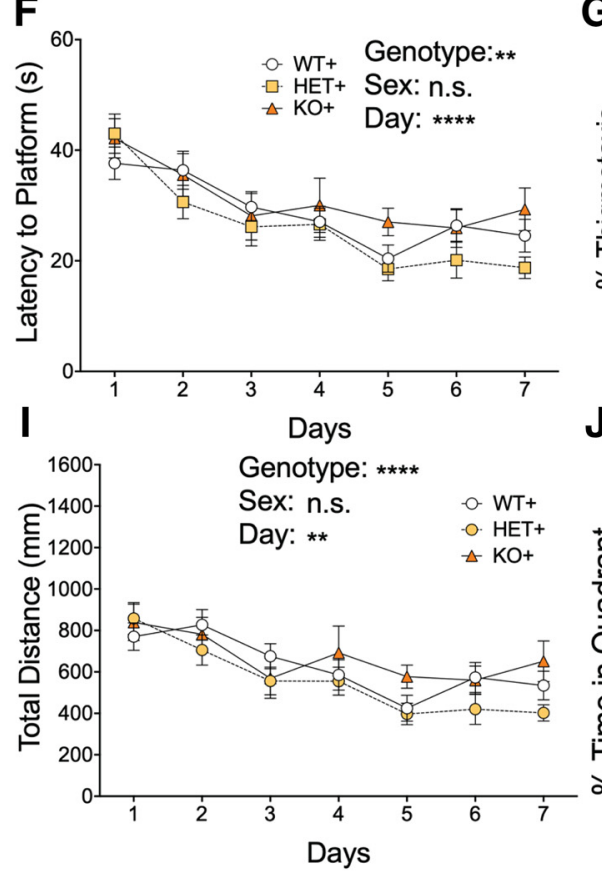
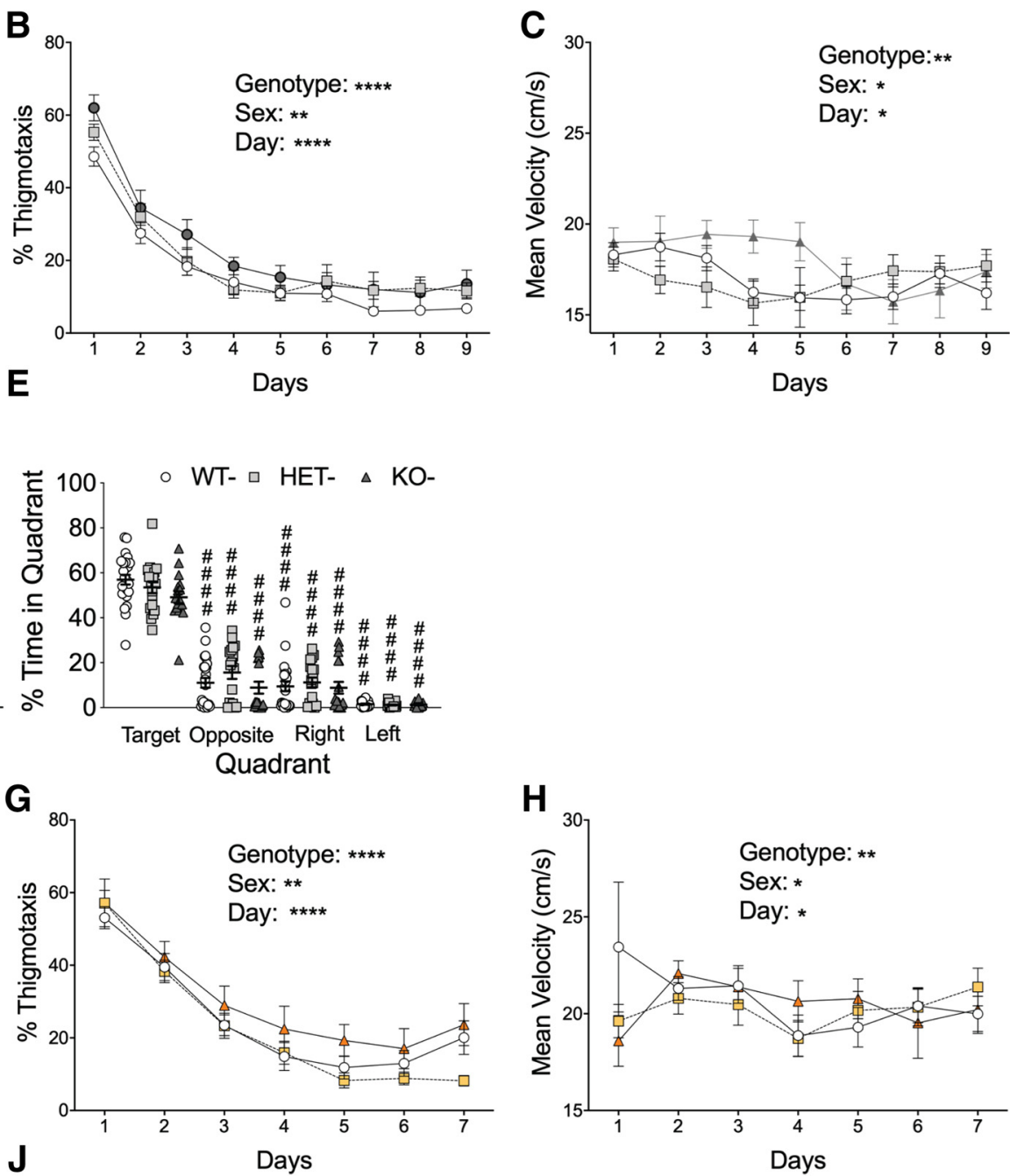

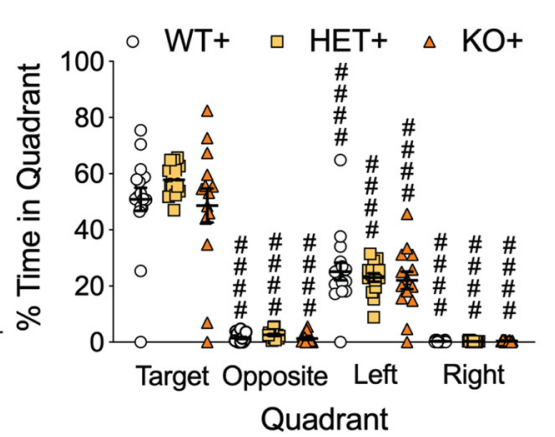

Figure 4. No alteration in spatial learning behavior in Shank3+ mutant mice. Shank3- mice all displayed similar levels of latency to reach the platform $(\boldsymbol{A})$, percent time in the thigmotaxis region of the maze $(\boldsymbol{B})$, mean velocity $(\boldsymbol{C})$, and total distanced traveled $(\boldsymbol{D})$ during the training phase of the Morris water maze test. In the probe trial, all three groups showed a preference for the target quadrant compared with other quadrants $(\boldsymbol{E})$. Shank3+ mice all displayed similar levels of latency to reach the platform $(\boldsymbol{F})$, percent time in the thigmotaxis region of the maze $(\boldsymbol{G})$, mean velocity $(\boldsymbol{H})$, and total distanced traveled $(\boldsymbol{I})$ during the training phase of the Morris water maze test. $\boldsymbol{J}$, In the probe trial, all three groups showed preference for target quadrant. Data represented as mean \pm SEM; \#\#\#\# $<0.0001 ; n=24 \mathrm{WT}-, n=16 \mathrm{HET}-, n=20 \mathrm{KO}-, n=24 \mathrm{WT}+, n=19 \mathrm{HET}+, n=16 \mathrm{KO}+$.

based on previous studies (Chen et al., 1998), we expected this NSE-tTA transgene to express tTA largely limited to the striatum and cerebellum. Unfortunately, our data revealed a much more widespread NSE-tTA expression based on rescue of our Shank3 ${ }^{\mathrm{E} 13}$ mutant (Fig. 1).
Second, based on many published reports including our own (Monteggia et al., 2004), we anticipated that breeding and rearing mice on doxycycline-containing water would successfully suppress tTA activation of cre-recombinase expression so that we might use doxycycline withdrawal 
A

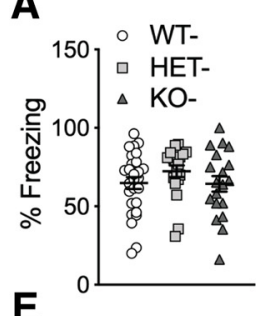

-O WT-

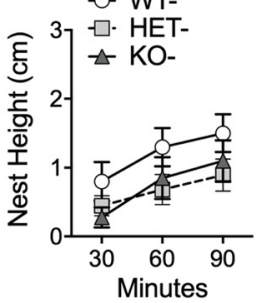

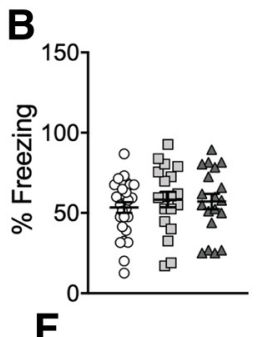

$\mathbf{F}$

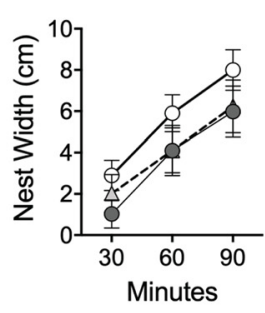

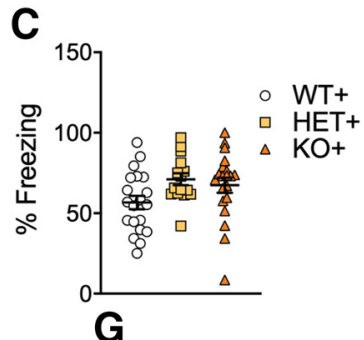

-o- WT+

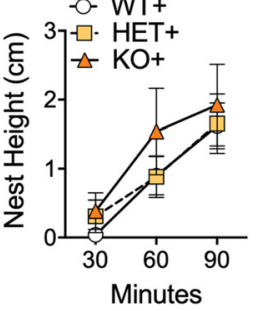

- $W T+\mathbf{K}$

- WT+

$\triangle \mathrm{KO}+$

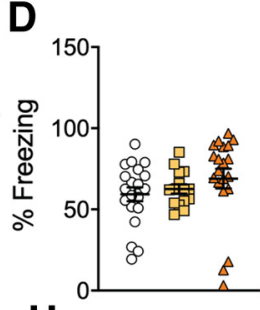

H

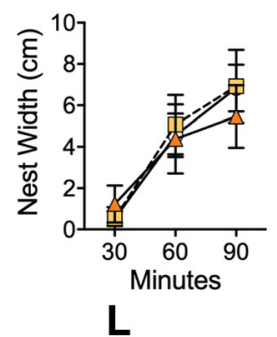

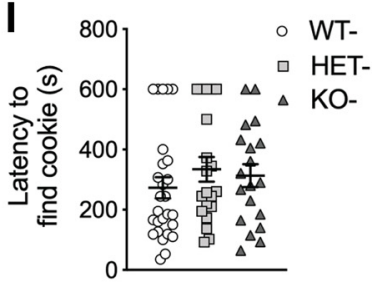
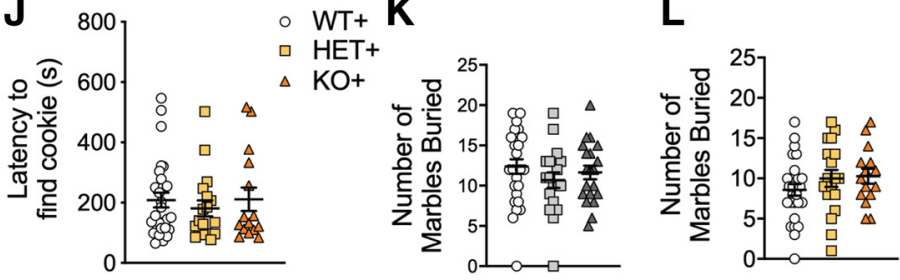

Figure 5. No phenotypes associated with fear conditioning and innate behaviors in Shank3- and Shank3+ mice. In both the cue (A) and contextual $(\boldsymbol{B})$ fear conditioning test, control mice spent similar percent freezing. Similarly, Shank3+ mice displayed similar percent freezing in cue $(\boldsymbol{C})$ and contextual $(\boldsymbol{D})$ fear conditioning. In nesting behavior Shank3- control mice displayed similar nest heights $(\boldsymbol{E})$ and widths $(\boldsymbol{F})$. Similarly, Shank3+ mice displayed similar nest heights $(\boldsymbol{G})$ and widths $(\boldsymbol{H})$. In the palatable treat-finding test, there were no difference observed between Shank3- mice groups (I) and Shank3+ mice groups (J), respectively. Additionally, Shank3mice buried similar number of marbles among groups $(\boldsymbol{K})$ and well as Shank3+ mice groups $(\boldsymbol{L})$. Data represented as mean $\pm \mathrm{SEM}$; $* p<0.05 ; * * p<0.01, * * * p<0.001, * * * * p<0.0001 ; n=24 \mathrm{WT}-, n=16 \mathrm{HET}-, n=20 \mathrm{KO}-, n=24 \mathrm{WT}+, n=19 \mathrm{HET}+, n=16 \mathrm{KO}+$.

to regulate the temporal onset of genetic reversal by crerecombinase. Our data using doxycycline revealed that the cre-recombinase expression was "leaky" and allowed for genetic reversal of Shank3 ${ }^{\mathrm{E} 13}$ to WT Shank3 even when doxycycline was provided (data not shown). Thus, we were only able to examine early developmental genetic rescue using this strategy.

\section{Social interaction tests in Shank3 mutant mice}

Considering that social deficits are a core feature of autism and a prominent feature of PMS, we tested Shank3 mutant mice in social tasks. We assessed reciprocal social interaction by pairing mice of the same genotype and sex with one another for an initial encounter. Shank3 KOmice displayed a significant decrease in social interaction compared with HET- mouse pairs (total interaction: WTvs KO-: $p=0.0508$; HET- vs KO-: $p=0.012$; WT- vs HET-: $p=0.750$; Fig. $2 A$ ) a result that replicates our previous findings in Shank3 ${ }^{\mathrm{E} 13} \mathrm{KO}$ mice. We did not find a significant difference in social interaction between WT- and KO- mice compared with our previous study (Jaramillo et al., 2017), however, there was a trend toward significance $(p=0.0508)$ between WT- and KO- mice. In our genetically reversed Shank3 cohort $(\mathrm{WT}+, \mathrm{HET}+, \mathrm{KO}+)$, all three groups of mice displayed similar interaction times (Fig. 2B). These data indicate decreased reciprocal social interaction in Shank3 KO mice compared with Shank3 HET mice that was rescued with genetic reversal.

We next tested mutant mice in a social recognition memory task in which novel juvenile mice (three weeks of age) were introduced to experimental mice, then $3 \mathrm{~d}$ later the same juvenile mice were reintroduced to the same experimental mouse. Shank3 WT- mice displayed a significant decrease in interaction time when the juvenile mouse was reintroduced, suggesting WT- mice displayed social recognition memory. Similar to our previously published findings, both HET- and KO- mice displayed a lack of social recognition memory (initial vs recognition time, WT-: $p=0.002$, HET-: $p=0.317$, KO-: $p=0.373$; Fig. $2 C$ ). In our genetically reversed Shank3 mice WT+, HET+, and KO+ mice all displayed significant levels of social recognition memory between the initial and recognition trials (initial vs recognition time, WT+: $p=0.024, \mathrm{HET}+: p=0.04, \mathrm{KO}+$ : $p=0.019$; Fig. $2 D$ ), suggesting effective genetic rescue of social recognition memory.

In our previous study, Shank ${ }^{\mathrm{E} 13}$ HET and KO mutant mice displayed abnormal social novelty exploration with HET mice also showing abnormalities in social versus inanimate preference in the three-chamber test of sociability (also known as three-box social interaction test; Jaramillo et al., 2017). We replicated these findings in the present study comparing Shank3 HET- and KO- mutant mice to WT- littermates (mice lacking at least one of the 
two transgenes needed for genetic rescue). In the initial trial to detect any baseline chamber bias, none of the groups displayed a bias (Fig. 2E). However, when one chamber contained a novel caged adult mouse and the other chamber contained an inanimate object, only the WT- and KO- mice displayed a preference for the social target. Replicating our previously published results (Jaramillo et al., 2017), the Shank3 HET- mice did not display significant sociability or preference for the social target (social vs inanimate, WT: $p<0.00,001$; HET-: $p=0.196$, KO-: $p<0.0012$; Fig. 2F). Additionally, when one of the chambers contained a familiar caged adult mouse, and the other chamber contained a novel caged adult mouse, only WT mice preferred the novel social target. Shank3 HET- and KO- mice did not display a preference for social novelty (novel vs familiar WT-: $p<0.014$, HET-: $p=-0.703$, KO-: $p=0.181$; Fig. $2 G$ ).

In our genetically reversed Shank3+ mice (containing both transgenes required for genetic rescue), all three groups of mice (WT+, $\mathrm{HET}+, \mathrm{KO}+)$ displayed no initial chamber bias in the initial trial of the three-chamber test (Fig. $2 H$ ). In the social versus inanimate preference trial, both $\mathrm{WT}+$ and $\mathrm{KO}+$ mice displayed a preference for the social target, however, genetically rescued Shank3 HET+ mice still did not display a social preference (social vs inanimate WT+: $p<0.0044$; HET+: $p=0.153, \mathrm{KO}+$ : $p=0.042$; Fig. $2 /$ ). In the social novelty trial, WT + and $\mathrm{KO}+$ mice preferred the novel social target. Although there was a trend toward preference for the novel target in HET + mice, it was not significant. Thus, HET+ mice did not display a preference for social novelty (novel vs familiar WT $+: p=0.017$, HET $+: p=0.099, \mathrm{KO}+: p=0.037$; Fig. $2 J)$. These findings replicate a lack of sociability in Shank3 HET mice and indicate that this particular deficit unique to heterozygotes was not reversible by genetic rescue of SHANK3 expression. Lastly, using a different social approach task referred to as "caged conspecific," time spent approaching a novel mouse in an OF did not differ among genotypes (Fig. $2 K, L$ ), representing a failure to replicate the difference previously observed in homozygous Shank3 ${ }^{\mathrm{E} 13}$ mice (Jaramillo et al., 2017).

\section{Restricted repetitive behaviors in Shank3 mutant mice}

Restricted and repetitive behaviors are another core feature of autism and PMS; thus, we monitored repetitive grooming behavior in Shank3 mutant mice over a $10 \mathrm{~min}$ period. In our previous study (Jaramillo et al., 2017), Shank ${ }^{\mathrm{E} 13}$ HET and KO mice displayed increase grooming. In this study, we replicated these findings in both the HET- and KO- mutant mice. Both genotypes displayed a significant increase in time spent grooming compared with WT- littermates (WT- vs HET-: $p=0.0004$; WT- vs KO-: $p=0.0007$; HET- vs KO-: $p=0.968$; Fig. $3 A$ ). Additionally, the increase in grooming time was accompanied by an increased number of grooming bouts in KOmice (WT- vs KO-: $p=0.030$, WT- vs HET-: $p=0.66$, HET- vs KO- $p=0.265$; Fig. $3 B$ ). Genetically reversed Shank3 mutant mice displayed similar grooming durations (Fig. $3 C$ ) and bouts (Fig. $3 D$ ) across all three genotypes, again suggesting complete rescue of the grooming phenotype.

\section{Anxiety-like behavior tests in Shank3 mutant mice}

Like our previous publication, we found no changes in anxiety-related behaviors in the mutant and no changes with genetic reversal as expected. We examined multiple tasks of relevance to anxiety. In the OF test, all three Shank3- genotypes (WT-, HET-, and KO-) spent similar amounts of time in the central, anxiogenic region (Fig. 3E). Additionally, there were similar distances traveled among the three groups in both the center and thigmotaxis region during the OF test (Fig. $3 F$ ). The genetically reversed mice also displayed similar durations in the center of the OF (Fig. 3G) and similar total distances traveled in both the center and thigmotaxis regions (Fig. $3 H$ ).

In the dark/light test, WT-, HET-, and KO- all displayed a similar latency to enter the anxiety-provoking, light chamber (Fig. 3/). However, KO- mice spent significantly less time in the light chamber when compared with HETmice (KO- vs HET-: $p=0.032$, WT- vs HET-: $p=0.300$, WT- vs KO- $p=0.395$ ) and significantly more time in the dark chamber when compared with HET- mice (KO- vs HET-: $p=0.032$, WT- vs HET-: $p=0.300$, WT- vs KO$p=0.395 ;$ Fig. 3J). In the genetically reversed Shank3 mice, $\mathrm{WT}+, \mathrm{HET}+$, and $\mathrm{KO}+$ all displayed similar durations in latency to enter the light side (Fig. $3 K$ ) and duration in the light and dark chambers, respectively (Fig. $3 L$ ). In the elevated plus maze test, WT-, HET-, and KO- all displayed similar durations in the closed and open arms (Fig. 3M). The genetic reversal Shank3 mice, WT+, HET+, and $\mathrm{KO}+$, also displayed similar durations in the closed and open arms (Fig. $3 N$ ).

Rearing activity in mice is thought to be a form of exploratory activity that could be linked to anxiety. In our previous studies, Shank $3^{\mathrm{E} 13} \mathrm{HET}$ and KO mice displayed decreased rearing events compared with WT mice (Jaramillo et al., 2017). We replicated these findings in Shank3 HET- and KO- mice. Both displayed a significant reduction in rearing events over a 30 min period compared with WT- mice (WT- vs HET-: $p=0.0004$, WT- vs KO-: $p=0.0002$, HET- vs KO-: $p=0.997$; Fig. 3O). In the genetically reversed mice, $\mathrm{WT}+, \mathrm{HET}+$, and $\mathrm{KO}+$ all displayed similar levels of rearing activity (Fig. 3P), again demonstrating successful genetic reversal of this phenotype.

In the locomotor test, Shank3 HET- and KO- mice displayed a significant reduction in locomotor activity over a 2-h time span compared with WT- mice (WT- vs HET-: $p>0.00,001$, WT- vs KO-: $p>0.00,004$, HET- vs KO-: $p>0.0006$; Fig. $3 Q$ ) consistent with our previous findings (Jaramillo et al., 2017). In the genetically reversed Shank3 mice, a main effect of genotype was also observed, though HET + mice did not show a reduction in locomotor activity compared with WT, suggesting possible reversal (Fig. 3R). Lastly, we tested for motor abnormalities using the rotarod test, and observed no significant difference in latency to fall among WT-, HET-, and KO- mice (Fig. 3S) or among WT+, HET+, and $\mathrm{KO}+$ mice, respectively (Fig. 
$37)$. Thus, we failed to replicate our original finding of incoordination on the rotarod in the Shank $3^{\mathrm{E} 13}$ mice (Jaramillo et al., 2017).

\section{Shank3 mice and spatial learning}

Recent studies by the CDC suggest $\sim 38 \%$ of children with ASD have intellectual disability (Christensen et al., 2018), the prevalence of which is even higher in PMS patients (Phelan and Rogers, 1993). Thus, we tested spatial learning and memory ability in the Shank3 mutants using the Morris water maze. Unlike our previously published data, we did not find any major difference in spatial learning/memory in Shank3 mutant mice. Although some main effects of genotype were observed statistically, the valence of such effects were not readily ascertained from the data. All mice in the control group showed similar latencies to reach the platform during the training phase of the test (Fig. 4A). Additionally, control mice showed similar percent time in the thigmotaxis zone (Fig. 4B), similar mean velocities (Fig. 4C), and similar distances traveled (Fig. 4D). In the probe trial, all three control genotypes displayed similar preference for the target quadrant versus all other quadrants (Fig. 4E). Genetically reversed Shank3 mice also displayed similar times in latencies to the platform (Fig. 4F), percentage of time in the thigmotaxis zone (Fig. 4G), mean velocities (Fig. 4H), and total distances traveled (Fig. 4/). In the probe trial all three genotypes displayed a preference for the target quadrant compared with all other quadrants (Fig. 4J). This represents a failure to replicate our previous finding of altered spatial learning and memory in Shank $3^{\mathrm{E} 13}$ homozygous mutants (Jaramillo et al., 2017).

\section{Assessing fear conditioning and innate behaviors in Shank3 mutant mice}

Studies in ASD have shown some patients display difficulties updating associations between environmental cues and aversive unconditioned stimuli (South et al., 2012). Thus, we used fear conditioning to test mice for abnormalities in associating a stimulus with aversive consequences. In both cue (Fig. 5A) and contextual (Fig. 5B) fear conditioning tests, WT-, HET-, and KO- mice displayed similar levels of freezing. Similarly, in the genetically reversed Shank3 mice, $\mathrm{WT}+, \mathrm{HET}+$, and $\mathrm{KO}+$ displayed similar levels of freezing in cue (Fig. $5 C$ ) and contextual (Fig. 5D) fear conditioning. This is the first study of fear conditioning in our Shank3 ${ }^{\mathrm{E} 13}$ mice to date.

Next, we assessed innate behaviors such as nest building and food finding. In the nest-building task, the height and width of nests built over a 90-min time period were assessed. Both control (Fig. 5E,F) and genetically reversed (Fig. 5G,H) mice displayed similar levels of nest height and width, respectively, among their genotypes. This is the first test of nest building in our Shank3 ${ }^{\mathrm{E} 13}$ mice to date.

In the food finding test, we buried a palatable treat with home bedding and measured their latency to find the hidden treat. WT-, HET-, and KO- mice displayed similar latencies to find a hidden treat (Fig. 5/). Similarly, WT+,
$\mathrm{HET}+$, and $\mathrm{KO}+$ mice displayed similar latencies to find the treat (Fig. 5J).

Lastly, in the marble burying task, Shank3 WT, HET, and $\mathrm{KO}$ mice in both cohorts buried similar numbers of marbles (Fig. $5 K, L$ ). These negative results in marble burying represent a failure to replicate our previous findings in Shank3 ${ }^{\mathrm{E} 13}$ mutants (Jaramillo et al., 2017).

\section{Discussion}

Shank3 is among the most well-characterized ASD-associated genes. Genetic analysis in ASD patients has shown there are over 40 known mutations throughout the Shank3 gene that either lead to truncating variants or are predicted to be deleterious (Jiang and Ehlers, 2013; Mameza et al., 2013; Leblond et al., 2014). Previously we showed that targeted disruption of the PDZ domain in the Shank3 gene in mice led to a number of behavioral abnormalities (Jaramillo et al., 2017). Mutant mice displayed repetitive grooming, social deficits, rotarod deficits, decrease rearing activity, and learning and memory deficits. These findings validated many findings in a previously published, similar Shank3 mouse model (Peça et al., 2011).

It is important to note that our genetic approach, based on previous studies (Chen et al., 1998) using this NSE-tTA transgene, was initially expected to limit tTA expression largely to the striatum and cerebellum. Unfortunately, our data revealed a much more widespread NSE-tTA expression based on rescue of our Shank3 ${ }^{\mathrm{E} 13}$ mutant (Fig. 1). We are currently unable to explain this discrepancy other than to suggest that promotor-driven transgenes can vary in their expression based on genetic background, and action of cre may well vary depending on the floxed gene of interest, in this case Shank $3^{\mathrm{E} 13}$. Second, based on many published reports including our own (Monteggia et al., 2004), we anticipated that breeding and rearing mice on doxycycline-containing water would successfully suppress tTA activation of cre-recombinase expression so that we might use doxycycline withdrawal to regulate the temporal onset of genetic reversal by cre-recombinase. Our data using doxycycline revealed that the cre-recombinase expression was leaky and allowed for genetic reversal of Shank3 ${ }^{\mathrm{E} 13}$ to WT Shank3 even when doxycycline was provided (data not shown). Thus, we were only able to examine early developmental genetic rescue using this strategy.

Thus, we set out to address the possibility that early genetic restoration of Shank3 may prevent the onset of the ASD-like behaviors. In combination with two other transgenic mouse lines (tetO-Cre and NSE-TTA), we generated a novel Shank $3^{\mathrm{E} 13}$ mutant mouse model in which a floxed, transcriptional stop cassette inserted into intron 12 of the Shank3 gene was removed early in development. We replicated many, but not all, previously published behavioral abnormalities in the control cohort, and observed restoration of SHANK3 expression and reversal of most of the replicated behavioral differences in Shank $3^{\mathrm{E} 13}$ mice following early genetic reversal.

Mutant KO- mice did not display preference for social novelty, while genetically reversed $\mathrm{KO}+$ mice displayed 
appropriate preference for social novelty in the three-box social interaction test. Similarly, mutant HET- and KOmice failed to demonstrate social recognition memory, while both genetically reversed $\mathrm{HET}+$ and $\mathrm{KO}+$ mice displayed normal social recognition memory.

In the genotype/sex-matched test of reciprocal social interaction, HET- mice showed a significant decrease in social interaction compared with WT-, partially replicating our previous findings (Jaramillo et al., 2017). Early genetic reversal of Shank $3^{\mathrm{E} 13}$ mutation ameliorated this difference in social interaction in the HET+ mice.

In one additional social domain task, we failed to replicate our previously published deficits in mutant KO- cohorts. The caged conspecific social approach test (also called social interaction with a caged adult) did not reveal differences among HET- or KO- and WT-, whereas previously we observed a decrease in the homozygous mutants (Jaramillo et al., 2017). This finding underscores the critical importance of running parallel control cohorts with genetic or pharmacologic reversal experiments. Not doing so may lead to a lack of significant difference being falsely interpreted as rescue of a previously demonstrated behavioral deficit.

We also demonstrated successful genetic rescue of both increased grooming and decreased rearing in our genetically reversible Shank3 mice. Overall, most behavioral abnormalities demonstrated in our mutant control cohorts were successfully rescued with genetic reversal.

While conducting our study, another group successfully generated and published a similar, genetically reversible Shank3 mutant model involving adult restoration of Shank3 in a Shank3 mutant mouse and showed rescue of social deficits and repetitive grooming behavior in homozygous Shank3 mutant mice (Mei et al., 2016). Mei et al., also showed the inability to rescue anxiety and motor deficits in adult Shank3 KO mice. While we did see a similar decrease in the locomotor activity in Shank3+ KO mice in our study, it was apparently only rescued in the heterozygous Shank3E13 mice. While both studies observed a rescue of ASD-like behaviors, the studies differ on the time points of genetic reversal (Feng et al.: P20-P21 and 2-4.5 months; this study: E18). Both studies replicate genetic reversibility of Shank3 deficits across similar tests and should be viewed as complementary. However, our study behaviorally characterized genetic rescue of the more clinically accurate heterozygous mutant mice. Following restoration of Shank3 expression Shank3+ heterozygous mice displayed a lack of repetitive grooming behavior and rescue of social interaction with a juvenile mouse but not a rescue of social preference or social novelty in the three-box social interaction test. The discrepancy in outcomes in social test is likely due to fundamental differences in what each test measures; social interaction with a juvenile mouse in an open arena is a test of reciprocal social interaction whereas the other two tests measure preference for one caged social versus an inanimate or novel target. Also, the age of the stimulus mouse and accessibility of the stimulus mouse differs among the two tests.
Overall, our studies suggest early genetic rescue as a potential genetic therapy for ASD-like behaviors in ASD associated with SHANK3 deletion/mutation. Taken together with previously published studies, genetic intervention in SHANK3-related ASD may be most effective earlier in development.

\section{References}

Alouani S, Ketchum S, Rambosson C, Eistetter HR (1993) Transcriptional activity of the neuron-specific enolase (NSE) promoter in murine embryonic stem (ES) cells and preimplantation embryos. Eur J Cell Biol 62:324-332.

Blundell J, Tabuchi K, Bolliger MF, Blaiss CA, Brose N, Liu X, Südhof TC, Powell CM (2009) Increased anxiety-like behavior in mice lacking the inhibitory synapse cell adhesion molecule neuroligin 2. Genes Brain Behav 8:114-126.

Blundell J, Blaiss CA, Etherton MR, Espinosa F, Tabuchi K, Walz C, Bolliger MF, Südhof TC, Powell CM (2010) Neuroligin-1 deletion results in impaired spatial memory and increased repetitive behavior. J Neurosci 30:2115-2129.

Bozdagi O, Sakurai T, Papapetrou D, Wang X, Dickstein DL, Takahashi N, Kajiwara Y, Yang M, Katz AM, Scattoni ML, Harris MJ, Saxena R, Silverman JL, Crawley JN, Zhou Q, Hof PR, Buxbaum JD (2010) Haploinsufficiency of the autism-associated Shank3 gene leads to deficits in synaptic function, social interaction, and social communication. Mol Autism 1:15.

Chen J, Kelz MB, Zeng G, Sakai N, Steffen C, Shockett PE, Picciotto MR, Duman RS, Nestler EJ (1998) Transgenic animals with inducible, targeted gene expression in brain. Mol Pharmacol 54:495503.

Christensen DL, Braun KVN, Baio J, Bilder D, Charles J, Constantino JN, Daniels J, Durkin MS, Fitzgerald RT, Kurzius-Spencer M, Lee LC, Pettygrove S, Robinson C, Schulz E, Wells C, Wingate MS, Zahorodny W, Yeargin-Allsopp M (2018) Prevalence and Characteristics of Autism Spectrum Disorder Among Children Aged 8 Years - Autism and Developmental Disabilities Monitoring Network, 11 Sites, United States, 2012. MMWR Surveill Summ 65:1-23

Costales JL, Kolevzon A (2015) Phelan-McDermid syndrome and SHANK3: implications for treatment. Neurotherapeutics 12:620630.

Dragatsis I, Zeitlin S (2001) A method for the generation of conditional gene repair mutations in mice. Nucleic Acids Res 29:E10.

Ebert DH, Greenberg ME (2013) Activity-dependent neuronal signalling and autism spectrum disorder. Nature 493:327-337.

Etherton MR, Blaiss CA, Powell CM, Südhof TC (2009) Mouse neurexin-1alpha deletion causes correlated electrophysiological and behavioral changes consistent with cognitive impairments. Proc Natl Acad Sci USA 106:17998-18003.

Forss-Petter S, Danielson PE, Catsicas S, Battenberg E, Price J, Nerenberg M, Sutcliffe JG (1990) Transgenic mice expressing beta-galactosidase in mature neurons under neuron-specific enolase promoter control. Neuron 5:187-197.

Guy J, Gan J, Selfridge J, Cobb S, Bird A (2007) Reversal of neurological defects in a mouse model of Rett syndrome. Science 315:1143-1147.

Jaramillo TC, Speed HE, Xuan Z, Reimers JM, Escamilla CO, Weaver TP, Liu S, Filonova I, Powell CM (2017) Novel Shank3 mutant exhibits behaviors with face validity for autism and altered striatal and hippocampal function. Autism Res 10:42-65.

Jiang YH, Ehlers MD (2013) Modeling autism by SHANK gene mutations in mice. Neuron 78:8-27.

Kouser M, Speed HE, Dewey CM, Reimers JM, Widman AJ, Gupta N, Liu S, Jaramillo TC, Bangash M, Xiao B, Worley PF, Powell CM (2013) Loss of predominant shank3 isoforms results in hippocampus-dependent impairments in behavior and synaptic transmission. J Neurosci 33:18448-18468. 
Kwon CH, Luikart BW, Powell CM, Zhou J, Matheny SA, Zhang W, Li Y, Baker SJ, Parada LF (2006) Pten regulates neuronal arborization and social interaction in mice. Neuron 50:377-388.

Leblond CS, Nava C, Polge A, Gauthier J, Huguet G, Lumbroso S, Giuliano F, Stordeur C, Depienne C, Mouzat K, Pinto D, Howe J, Lemière N, Durand CM, Guibert J, Ey E, Toro R, Peyre H, Mathieu A, Amsellem F, et al. (2014) Meta-analysis of SHANK mutations in autism spectrum disorders: a gradient of severity in cognitive impairments. PLoS Genet 10:e1004580.

Mameza MG, Dvoretskova E, Bamann M, Hönck HH, Güler T, Boeckers TM, Schoen M, Verpelli C, Sala C, Barsukov I, Dityatev A, Kreienkamp HJ (2013) SHANK3 gene mutations associated with autism facilitate ligand binding to the Shank3 ankyrin repeat region. J Biol Chem 288:26697-26708.

Mei Y, Monteiro P, Zhou Y, Kim JA, Gao X, Fu Z, Feng G (2016) Adult restoration of Shank3 expression rescues selective autistic-like phenotypes. Nature 530:481-484.

Mitz AR, Philyaw TJ, Boccuto L, Shcheglovitov A, Sarasua SM, Kaufmann WE, Thurm A (2018) Identification of 22q13 genes most likely to contribute to Phelan McDermid syndrome. Eur J Hum Genet 26:293-302.

Monteggia LM, Barrot M, Powell CM, Berton O, Galanis V, Gemelli T, Meuth S, Nagy A, Greene RW, Nestler EJ (2004) Essential role of brain-derived neurotrophic factor in adult hippocampal function. Proc Natl Acad Sci USA 101:10827-10832.

Moy SS, Nadler JJ, Perez A, Barbaro RP, Johns JM, Magnuson TR, Piven J, Crawley JN (2004) Sociability and preference for social novelty in five inbred strains: an approach to assess autistic-like behavior in mice. Genes Brain Behav 3:287-302.

Nadler JJ, Moy SS, Dold G, Trang D, Simmons N, Perez A, Young NB, Barbaro RP, Piven J, Magnuson TR, Crawley JN (2004) Automated apparatus for quantitation of social approach behaviors in mice. Genes Brain Behav 3:303-314.

Naisbitt S, Kim E, Tu JC, Xiao B, Sala C, Valtschanoff J, Weinberg RJ, Worley PF, Sheng M (1999) Shank, a novel family of postsynaptic density proteins that binds to the NMDA receptor/PSD-95/ GKAP complex and cortactin. Neuron 23:569-582.

Peça J, Feliciano C, Ting JT, Wang W, Wells MF, Venkatraman TN, Lascola CD, Fu Z, Feng G (2011) Shank3 mutant mice display autistic-like behaviours and striatal dysfunction. Nature 472:437442.

Phelan K, Rogers RC (1993) Phelan-McDermid syndrome. In: Gene reviews, (Pagon RA, Adam MP, Ardinger HH, Bird TD, Dolan CR, Fong CT, Smith RJH, Stephens K, eds). Seattle: University of Washington, Seattle.

Phelan MC (2008) Deletion 22q13.3 syndrome. Orphanet J Rare Dis 3:14.

Powell CM, Schoch S, Monteggia L, Barrot M, Matos MF, Feldmann N, Südhof TC, Nestler EJ (2004) The presynaptic active zone protein RIM1alpha is critical for normal learning and memory. Neuron 42:143-153.

South M, Newton T, Chamberlain PD (2012) Delayed reversal learning and association with repetitive behavior in autism spectrum disorders. Autism Res 5:398-406.

Tabuchi K, Blundell J, Etherton MR, Hammer RE, Liu X, Powell CM, Südhof TC (2007) A neuroligin-3 mutation implicated in autism increases inhibitory synaptic transmission in mice. Science 318:71-76.

Tachibana Y, Miyazaki C, Ota E, Mori R, Hwang Y, Kobayashi E, Terasaka A, Tang J, Kamio Y (2017) A systematic review and meta-analysis of comprehensive interventions for pre-school children with autism spectrum disorder (ASD). PLoS One 12: e0186502.

Tu JC, Xiao B, Naisbitt S, Yuan JP, Petralia RS, Brakeman P, Doan A, Aakalu VK, Lanahan AA, Sheng M, Worley PF (1999) Coupling of mGluR/Homer and PSD-95 complexes by the Shank family of postsynaptic density proteins. Neuron 23:583592.

Wang X, McCoy PA, Rodriguiz RM, Pan Y, Je HS, Roberts AC, Kim CJ, Berrios J, Colvin JS, Bousquet-Moore D, Lorenzo I, Wu G, Weinberg RJ, Ehlers MD, Philpot BD, Beaudet AL, Wetsel WC, Jiang YH (2011) Synaptic dysfunction and abnormal behaviors in mice lacking major isoforms of Shank3. Hum Mol Genet 20:30933108.

Yang M, Bozdagi O, Scattoni ML, Wöhr M, Roullet FI, Katz AM, Abrams DN, Kalikhman D, Simon H, Woldeyohannes L, Zhang JY, Harris MJ, Saxena R, Silverman JL, Buxbaum JD, Crawley JN (2012) Reduced excitatory neurotransmission and mild autism-relevant phenotypes in adolescent Shank3 null mutant mice. J Neurosci 32:6525-6541. 